\title{
Le livre d'Anaximandre et la société de Milet
}

In: Mètis. Anthropologie des mondes grecs anciens. Volume 11, 1996. pp. 37-68.

\section{Résumé}

Le livre d'Anaximandre et la société de Milet (pp. 37-68)

En partant de l'idée que la prose poétique qu'emploie Anaximandre implique la recherche d'un public de concitoyens et, plus largement, de contemporains, on analyse l'histoire de Milet au Vème siècle pour identifier ces lecteurs-autiteurs. Leur existence est démontrée par la constatation que les cartes milésiennes demandaient, pour être comprises, d'être accompagnées d'une explication verbale. Puis, on examine le fragment d'Anaximandre dans le contexte de la crise sociale milésienne et du droit archaïque. En conclusion, on suggère que deux réformateurs, Téléclès et Hippodamos, dont Aristote expose les idées, présentent des traits idéologiques et politiques fortement analogues à ceux qu'on trouve chez Anaximandre.

Citer ce document / Cite this document :

V. Garcia Quintela Marco. Le livre d'Anaximandre et la société de Milet. In: Mètis. Anthropologie des mondes grecs anciens. Volume 11, 1996. pp. 37-68.

doi : $10.3406 /$ metis.1996.1046

http://www.persee.fr/web/revues/home/prescript/article/metis_1105-2201_1996_num_11_1_1046 


\section{LE LIVRE D'ANAXIMANDRE ET LA SOCIÉTÉ DE MILET}

\section{A la mémoire de Carlos Alonso del Real}

Dans sa compilation des plus anciens philosophes de la bibliothèque du Lycée qu'il fait en suivant les directives de lecture d'Aristote ${ }^{1}$, Théophraste exprime parfois son point de vue sur le contenu des livres ou, du moins, de certaines phrases qu'il a lues et qu'il présente au cours de son exposé.

Ainsi, il affirme qu'Héraclite a laissé ses écrits inachevés à cause de sa mélancolie. Il dit qu'Anaximandre s'exprime en termes assez poétiques et qu'Anaximène avait un style épuré en langue ionienne ${ }^{2}$. Ce genre de commentaircs littéraires était généralisé chez les intellectuels grecs. Hécatée et Hérodote se moquent des efforts de leurs prédécesseurs, qu'ils considèrent puérils ou inexacts ${ }^{3}$. Platon/Socrate se plaint du décalage entre le contenu du livre d'Anaxagore et son titre ou préface. ${ }^{4}$. De même Aristote considère qu'Héraclite aurait dâ s'appliquer en ce qui concerne la ponctuation $^{5}$. Pour sa part Cicéron pense que la prose de Démocrite était clairement destinée à la communication orale ${ }^{6}$.

1. J.B. McDiarmid, «Theophrastus on the Presocratic Causes», Harv. St. Cl. Philol., 61, 1953, pp. 85-156. Suit et développe H. Cherniss, Aristotle's Criticism of Presocratic Philosophy, Baltimore, 1935.

2. Héraclite: Diogène Laërce, IX 6, qui cite expressément Théophraste; Anaximandre: Diels-Kranz, 12 A 9, Simplicius vient aussi citer Théophraste; Anaximène: Diogène Laërce, II 3, sans référence explicite à Théophraste cette fois-ci.

3. Hécatée, FGrHist, $1 \mathrm{~F} \mathrm{1;} \mathrm{Hérodote,} \mathrm{IV} \mathrm{36,} \mathrm{commentés} \mathrm{par} \mathrm{M.} \mathrm{Detienne,}$ $L$ 'invention de la mythologie, Paris, 1981, pp. 91-103.

4. Phédon, 97c-99d (Diels-Kranz, 59 A 47) et voir infra.

5. In Rhétorique, III $51407 \mathrm{~b} 11$ (Diels-Kranz, 22 A 4) qui fait allusion au fragment Diels-Kranz, 22 B 1.

6. De oratione, I, 11,49 et 20,67 (Diels-Kranz, 68 A 34). 
Mais cela ne se limite pas à un seul courant intellectuel que nous pouvons appeler philosophique ou rationnel. A partir du passage à l'écrit de l'épopée traditionnelle tout au long de l'époque archaique, sa discussion, sa critique, son exégèse et sa correction ont conduit à l'établissement d'une morale en accord avec les situations très changeantes de ce que l'on a appelé l'époque des expériences de l'Histoire de la Grèce'. Héraclite, Xénophane de Colophon ou Platon ont élaboré des parts importantes de leur pensée en opposition à la tradition épique. Pythagore essaie de la récupérer à son avantage et Parménide fait peut-être de même ${ }^{8}$. En ce sens, l'invention du commentaire allégorique de la poésie que font les rhapsodes professionnels a une place analogue ${ }^{9}$. La position littéraire de Phérécyde de Syros a donné lieu à de nombreuses exégèses (voir infra).

Pour conclure, les anciens Grecs avaient parfaitement conscience de l'importance des formes qu'adoptait l'expression du savoir, de leur provenance et de la manière de les manipuler. Si Théophraste commente certains aspects de forme des livres d'Anaximandre ou Anaximène, c'est parce qu'il les considère pertinents et non pas seulement comme une démonstration d'érudition littéraire.

Or s'il est aisé d'accepter ce fait comme prémisse, il n'est pas aussi facile d'en tirer des conséquences significatives. En effet, une étude uniquement rhétorique des textes de la philosophie naissante, comme s'il s'agissait de formcs sans contenu, est-clle pertinente? Ou, au contraire. Peut-on comprendre les contenus sans tenir compte des nombreuses conditions matérielles qui les limitent? Je pense que la réponse est non dans les deux cas; de plus il faut poser d'une autre manière les questions auxquelles nous conduisent les données exposées.

La question qui me semble pertinente est la suivante: quand Anaximandre

7. A.M. Snodgrass, La Grèce archaïque, le temps des apprentissages, Paris, 1986 (1980), au sujet de la critique à l'époque archaïque G.E.R. Lloyd, Magic, Reason and Experience. Studies in the Origin and Development of Greek Science, Cambridge, 1979 , p. 10 et sqq.

8. Pour Pythagore voir M. Detienne, Homère, Hésiode et Pythagore. Poésie et philosophie dans le pythagorisme ancien, Bruxelles, 1962; pour Parménide, M.E. Pellikaan-Engel, Hesiod and Parmenides. A New View on their Cosmologies and on Parmenides Proem, Amsterdam, 1978.

9. G. Rocca-Serra, «Naissance de l'exégèse allégorique et naissance de la raison», in J.-F. Mattéi (éd.), La naissance de la raison en Grèce, Actes du congrès de Nice, mai 1987, Paris, 1990, pp. 77-82, remet en question la primauté traditionnellement admise de Théagène de Rhégium pour distinguer des personnages tels que Métrodore de Lampsaque et Stésimbrotos de Thasos. 
choisit la prose poétique comme style et le livre ou Jiv $\alpha \xi$ comme support à qui prétend-il s'adresser? La réponse que donne $\mathrm{Ch}$. $\mathrm{H}$. Kahn me semble insuffisante:

\begin{abstract}
«It was he [Anaximander] who first wrote down his views $\pi \varepsilon \rho i$ pú-

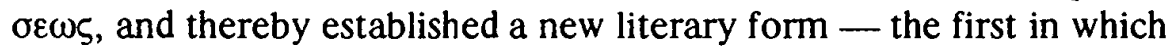
prose was employed - which was to serve as the written basis for the new scientific tradition. His work is thus the ancestor for all later specimens of the genre, including the Timaeus, the physical treatises of Aristotle, and the De rerum natura of Lucretius. It is this written work which made possible the preservation and transmission of Anaximander's views, their discussion by Aristote, their summary by Theophrastus, and, finally, their citation by Simplicius in his commentary on the Physics. It is, as it were, this act of Anaximander in committing his ideas to writing which brought the history of Greek philosophy into existence»:0.
\end{abstract}

Rien de cela n'est faux, mais rien ne prouve qu'Anaximandre pensait à la postérité quand il écrivait. Les effets à plus ou moins long terme des actes des individus sont une chose, la justification et/ou la genèse de ces actes dans une psyché donnée en sont une autre et leur effet plus ou moins immédiat dans le contexte de la société dont ils émanent et qui, présumablement, en est la première consommatrice ou usagère, une troisième.

Le texte de $\mathrm{Ch}$. H. Kahn et, en général, l'histoire traditionnelle de la philosophie rendent compte de la première situation: l'établissement d'une dialectique dans le temps entre des pensées logiquement enchaînées les unes aux autres par leur contenu. La deuxième approche implique la compréhension de l'individu et appartient au domaine de la psychologie historique; elle serait, en elle-même, la plus intéressante. Néanmoins, nous devons reconnaître que, dans le cas qui nous occupe, nous manquons de l'information suffisante pour élaborer cette analyse. La troisième approche est celle que nous développerons ici pour répondre à plusieurs questions: en choisissant des formes poétiques, à qui Anaximandre s'adressait-il? Sa pensée avait-elle un sens pour ses concitoyens? Son œuvre a-t-elle eu des

10. Ch. H. Kahn, Anaximander and the Origins of Greek Cosmology, New York, 1960, pp. 6-7. Néanmoins, dans un article sur Empédocle de la même année («Religion and Natural Philosophy in Empedocles' Doctrine of the Soul», Archiv fiur Geschichte der Philosophie, 42, 1960, pp. 3-35), Kahn établit la différence entre les deux poèmes d'Empédocle en fonction de leur public et de leur degré de communicabilité, spécialement pp. 27-30. 
échos dans sa cité? A chacune de ces questions correspond une partie de notre travail ".

\section{Milet et Anaximandre}

Pour situer Anaximandre et son public il faut nous intéresser à l'histoire de la cité de Milet. Or, dans cette histoire, ce n'est pas la véracité des faits, plus ou moins grande selon les cas, qui importe, mais le fait que les Milésiens, ou du moins des groupes ou secteurs déterminés de la cité, agissaient comme si les choses s'étaient vraiment passées comme cela.

En prenant en compte cette prémisse, on peut affirmer tout d'abord, que Milet est une colonie grecque établie dans la partie asiatique des côtes de la Mer Égée dont les habitants considéraient qu'ils. provenaient d'Athènes ou de Messénie (selon les diverses traditions et époques) ${ }^{12}$. Dans certains cas, comme autoaffirmation orgueilleuse, ils se considéraient autochtones, installés depuis toujours dans ce lieu ${ }^{13}$.

La première forme de gouvernement de la cité a été monarchique. Les rois étaient élus au sein d'une famille royale en fonction de leur excellence militaire ou religieuse. Cette institution fut abandonnée à cause d'une dispute à propos du meilleur candidat au trône à un moment donné. Plus concrètement, un roi élu fut tué par son adversaire qui instaura la tyrannie. Le fils et les amis du défunt se replièrent dans la forteresse d'Assèssos, dans l'intérieur du territoire milésien, et, avec l'aide des dieux, finirent par vaincre le tyran, sans toutefois restaurer la monarchie ${ }^{14}$.

11. Je ne prétends pas ainsi donner une explication globale de la pensée d'Anaximandre, car pour cela il faudrait inclure nécessairement une analyse de son absorption et réélaboration par l'imaginaire mythique religieux traditionnel. Il s'agit ici d'explorer sa dimension socio-politique à laquelle nous conduit le contrôle de l'audience de sa «prose poétique».

12. Examinées par M.B. Sakellariou, La migration grecque en Ionie, Athènes, 1958, pp. 39-65.

13. A. Momigliano, "Questioni di storia Ionica arcaica», in Quinto contributo alla storia degli studi classici e del mondo antico, Rome, 1975, pp. 369-402, (1933) surtout pp. 382-389. Visions plus récentes du caractère autochtone in E. Montanari, Il mito dell'autoctonia. Linee di una dinamica mitico-politica ateniese, Rome, 1981, avec une référence aux Ioniens p. 99 et sqq.; N. Loraux, Les enfants d'Athéna. Idées athéniennes sur la citoyenneté et la division de sexes, Paris, 1984, passim sur l'importance de ce mythe dans l'imaginaire politique athénien.

14. Conon, FGrHist, 26, F 1, xliv; Nicolas de Damas, FGrHist, 90 F 52-53; voir F. Ruzć, «Lc stylc ionicn dans la vie politique archaïque», Revue Et. Anc., 87, 1985, pp. 157-167, pp. 158-162. N. Robertson, «Government and Society at Miletus, 525-442 B.C.», Phoenix, 41, 1987, pp. 356-398, pp. 369-373, donne moins de fiabilité à l'épisode. De notre point de vue ce qui est fondamental c'est que ces histoires circulaient à Milet et formaient l'imaginaire social de ses habitants. 
La topographie du territoire de Milet explique le sens des gestes politiques implicites de cet épisode et d'autres qui lui sont postérieurs. En effet, les parties de ce territoire sont peuplées par des gens aux caractéristiques et aux ressources différentes et que symbolisent justement les traits différentiels du territoire de la cité qu'ils occupent. Ainsi il existe la cité de Milet proprement dite et ses alentours, vers l'intérieur s'étend la plaine avec le sanctuaire d'Apollon à Didymes et dans la périphérie on trouve des endroits comme Assèssos et Teichioussa qui sont limitrophes avec le territoire des indigènes cariens et présentent une nombreuse population d'origine locale; dans la mer, les îles de Lade, Leros et Lepsia sont aussi milésiennes ${ }^{15}$.

Si nous tenons compte de la topographie milésienne, nous constatons, dans l'épisode politico-institutionnel que nous venons d'évoquer, que la cité fondée par des Grecs arrivés par la mer retrouve son équilibre grâce à ceux qui s'étaient réfugiés dans l'intérieur anatolien. Cette dialectique d'influences grecques ou anatoliennes à grande échelle ou, dans une moindre mesure, de Milésiens tentés de tourner leur regard vers la Grèce ou vers l'Asie, préside à la vie politique et culturelle de la cité.

L'époque suivante sur laquelle nous avons des informations correspond à la tyrannie de Thrasybule. Laissons de côté diverses anecdotes sur ce personnage et soulignons ici sa situation très nette dans le schéma symbolico-spatial que nous venons de décrire. En effet, le roi lydien Alyattes attaqua Milet pendant onze ans, dévastant les cultures au moment de la récolte. Mais, malgré deux défaites terrestres, la cité résista au siège, grâce aux vivres envoyés par ses alliés de l'île de Chios et une faute religieuse commise par les Lydiens qui les obligea à rétablir la paix ${ }^{16}$.

Cet épisode présente clairement l'abandon des terres et des habitants de l'intérieur. Thrasybule se centre sur le noyau urbain et ses habitants protégés par leurs communications maritimes. Au gouvernement du tyran, qui finit aux alentours de 595-590, succèdent ceux de Thoas et Damasenor, dont on ne sait presque rien et qui laissent la place à une longue période d'instabilité sociale et politique qui dure jusqu'au début de la tyrannie d'Histiée vers 525 .

Anaximandre, qui avait soixante-quatre ans vers $547 / 6$ et mourut peu

15. La situation préhellénique, mais avec la même distribution, est évoquée par Homère, Iliade, II, 867-871; descriptions valables pour le VIème siècle in Hérodote, VI, 20. Assèssos, Hérodote, I, 19, 22, Robertson, art. cit., pp. 372-373. Teichioussa, SylP, 3d avec commentaire et $M$. Piérart, «Athènes et Milet II. L'organisation du territoire», Museum Helveticum, 42, 1985, pp. 276-299, pp. 287-292. Voir aussi M. Piérart, «Athènes et Milet I. Tribus et dèmes milésiens», Museum Helveticum, 40, 1983, pp. 1-18, pp. 12-13.

16. Hérodote, I, 17-22. 
après, a passé la plupart de sa vie dans cette situation. ${ }^{17}$ Vers le milieu du siècle les Milésiens s'adaptèrent sans problèmes au changement d'hégémonie politique en Anatolie da à la défaite des Lydiens et leur intégration dans l'empire perse, changement qui maintenait les conditions existant auparavant avec les Lydiens: paiement de tributs et aide militaire ${ }^{18}$.

Bien qu'ils proviennent de traditions différentes, les témoignages au sujet de la crise intérieure de Milet concordent. Deux d'entre eux désignent les

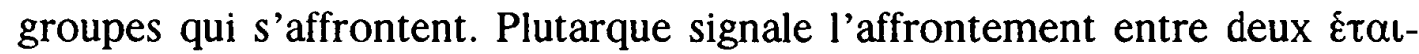

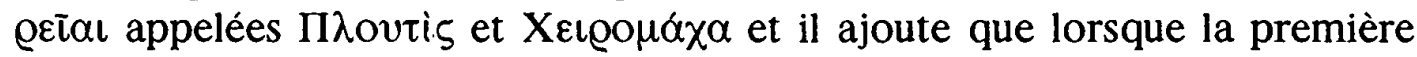
contrôlait la cité:

«ils délibéraient sur les affaires de première importance dans leurs navires et à une grande distance de la côte. Mais quand ils avaient pris

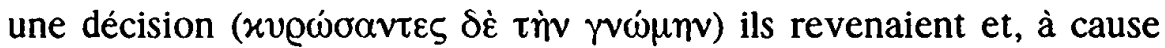

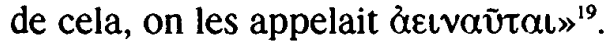

Pour sa part, Héraclide Pontique indique l'affrontement entre ceux qui

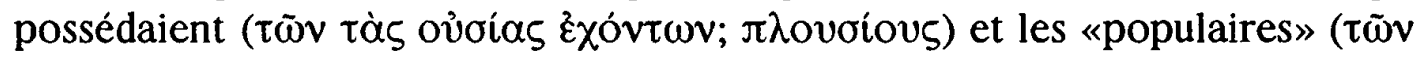

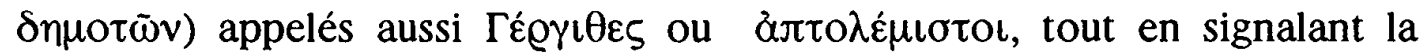
cruauté des deux groupes à l'heure de la victoire ${ }^{20}$.

Ainsi, les plus riches, qui obtenaient une part significative de leurs revenus de relations comerciales par voie maritime, formaient l'une des factions. Néanmoins la composition exacte de l'autre groupe est encore débattue. En effet, il peut s'agir d'agriculteurs indigènes qui avaient été soumis lors de la colonisation et qui donnaient des signes de vitalité politique, ou d'un groupe jouissant d'une certaine richesse obtenue de l'exploitation de la terre et qui formerait le noyau de l'infanterie des hoplites de Milet ${ }^{21}$.

17. Voir in M. Conche, Anaximandre. Fragments et Témoignages, Paris, 1991, pp. 30-35 un bref panorama de l'époque.

18. Hérodote, I, 141,$4 ; 143,1 ; 169,2$.

19. Plutarque, Questions grecques, 32.

20. Héraclide Pontique apud Athénée, XII, 524 a-b. Les Gergithes sont des paysans indigènes en Troade selon Hérodote, V, 122 et VII, 43.

21. Ruzé, art. cit., pp. 162-165 indique l'inexistence du démos en tant que tel, il n'existe pas de notion d'égalité. C. Talamo, «Isteo ed Erodoto. Per la storia della tirannide a Mileto», Rendiconti della accademia di Archeologia lettere e belle arti, Napoli, 44, 1969, pp 173-203, affirme que les Xeı@oúa $\chi \alpha$ sont des hoplites, pp. 186-

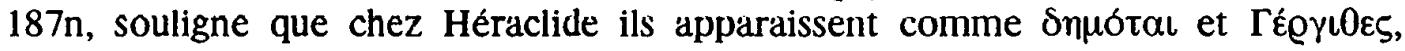
annulant ainsi le parallèle communément évoqué d'Hérodote, p. 187; de plus Apollon ne les citerait pas ainsi dans le texte de son oracle. Cependant Talamo ne

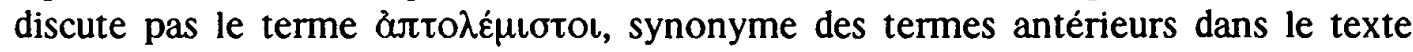
d'Héraclide et qui indique clairement l'absence de caractère guerrier. Robertson, art. 
En tout cas, les deux groupes se situent dans la dialectique traditionnelle de l'histoire milésienne avec leurs territoires d'opérations spécifiques

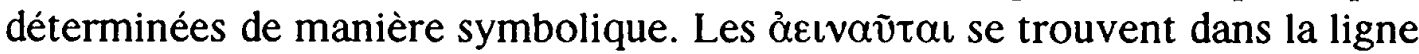
des fondateurs de la cité et des groupes qui triomphaient sous la tyrannie de Thrasybule, ils se tournent vers la mer et la tradition commerciale de la cité. Les «populaires» représentent le territoire de la $\chi \omega \dot{\varrho} \alpha$, les espaces cultivés de l'intérieur, présidés par les temple d'Apollon à Didymes et d'Athéna à Assèssos $^{22}$. Ils sont les héritiers symboliques des défenseurs de la monarchie légitime dont la base se situe dans la campagne. Peu importe que ce soient des indigènes hellénisés ou des Grecs de pure souche, ce qui importe c'est qu'ils s'intègrent dans la cité et déterminent leurs destins en s'affrontant aux premiers. Hérodote fait le récit de la fin de cette situation vers 525 av.J.C.:

«Milet... avait été victime de luttes internes des plus acharnées pendant deux générations, jusqu'au moment où les Pariens ont rendu l'ordre à la cité ... de la manière suivante: Quand les citoyens les plus

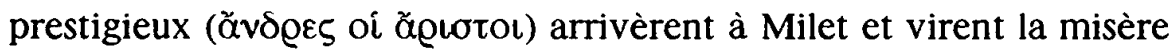
terrible dans laquelle se trouvait vraiment la population, ils manifestèrent leur désir de parcourir longuement leur pays $(\delta\llcorner\varepsilon \xi \varepsilon \lambda$ $\theta \varepsilon i v$ iǹ $\chi \omega \dot{g} \alpha v)$. Or, au cours de leur inspection, ils parcoururent tout le territoire de Milet; et, chaque fois qu'au milieu de la désolation qui régnait dans la $\chi \omega \dot{\omega} \alpha$, ils voyaient un champ bien cultivé, ils prenaient

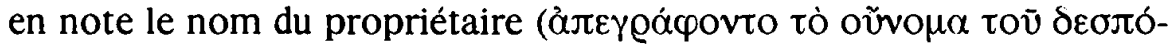

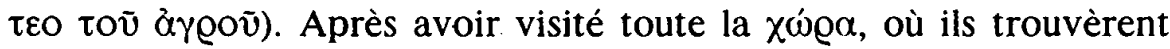
peu de champs présentant ces conditions, juste après leur retour à la

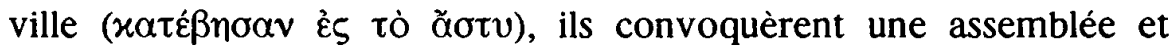
désignèrent, pour diriger la pólis, les personnes dont les champs étaient bien cultivés, car, selon leurs déclarations, ils considéraient que ces individus s'occuperaient des affaires de l'État ( $\tau \bar{\omega} v \delta \eta \mu o \sigma i ́ \omega v)$ avec autant de zèle que des leurs; et au reste des Milésiens, qui avaient été jusque-là la proie des luttes, ils leur ordonnèrent d'obéir aux autres ${ }^{23}$.

cit., pp. 374-375, défend avec solidité le point de vue traditionnel qui considère les $\Gamma \varepsilon ́$ pyı $\theta \varepsilon \varsigma$ comme des indigènes cariens. Le même auteur, pp. 381-4, interprète les

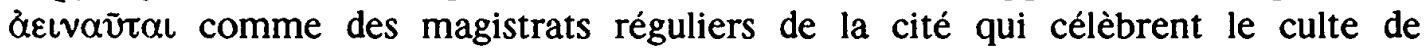
l'«embarquement» et suggère que son oligarchie doit coïncider avec celle qui est établie, selon Hérodote, en l'an 525.

22. In Hérodote, I, 147, les Ioniens prirent comme rois des Lyciens descendants de Glaucos. Assèsos est un site carien et la base des partisans du bon roi assassiné; il y avait là-bas un temple dédié à Athéna qu'Alyattes détruit pendant la guerre, destruction qui lui vaut un châtiment (supra n. 15).

23. Hérodote, V, 28-29. 
Du point de vue de l'histoire politique et sociale, ce récit présente des problèmes, car nous savons effectivement que, pendant la dernière partie du siècle, Milet était soumise aux Perses et que le gouvernement interne de la cité était aux mains de deux tyrans appelés Histiée et Aristagore. Mais il n'est pas nécessaire de voir une contradiction entre les deux épisodes. Sous n'importe quel régime politique les Grecs ont toujours aimé s'identifier à leurs personnages importants ou à leurs porte-paroles qui apparaissent souvent sans une localisation institutionnelle clairement définie. Ainsi Thucydide présente Périclès dans la démocratie athénienne comme un gouvernant unique malgré les formes démocratiques ${ }^{24}$, Hérodote et Aristote signalent que le tyran Pisistrate exerçait le pouvoir sans pour autant altérer les institutions de Solon ${ }^{25}$, alors que d'autres sources parlent de tyrans au pluriel, sans prendre l'expression comme un terme institutionnel, mais pour désigner des groupes dominants dans diverses cités ${ }^{26}$.

Ainsi, Milet a pu être gouvernée par une oligarchie réduite d'origine agricole, à la tête de laquelle se trouvaient des amis des Perses ${ }^{27}$ que la tradition historique grecque présente comme des tyrans, sans entrer dans leur localisation précise dans le cadre institutionnel de la cité. Passons, donc, à l'examen plus approfondi du geste des Pariens et de la solution donnée au conflit pour pouvoir ainsi éclaircir la position d'Anaximandre dans sa cité.

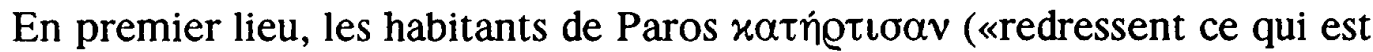
abîmé»), de la même manière, plus tard, le tyran Histiée demande à la Cour

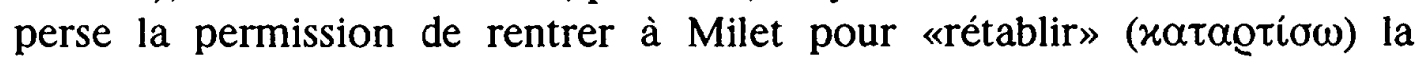
situation $^{28}$. Démonax de Mantinée fait de même quand il se charge d' «arranger» la situation institutionnelle de Cyrène ${ }^{29}$. Il s'agit d'examiner la situation et de la résoudre du mieux possible, bien que les solutions apportées soient différentes dans chacun des trois $\operatorname{cas}^{30}$. Le terme employé

24. Thucydide, II, 65.

25. Hérodote, I, 59,6; Aristote, Constitution des Athéniens, 16, 8-9; selon Aristote, à Milet, la tyrannie émanait des prytanes, Politique, V, 5, 1305 a 15-18.

26. S. Mazzarino, Fra Oriente e Occidente. Ricerche di storia Greca arcaica, Florence, 1947, pp. 231-232.

27. Les Perses appuient ce groupe, car, comme il garantit la dépendance agricole de la cité, ils évitent une situation comme celle qui se produisit auparavant lorsque, du continent, il leur était impossible de dominer Thrasybule (voir Talamo, art. cit., pp. 194-197).

28. Hérodote, V, 106, 5 .

29. Hérodote, IV, 161.

30. Arbitrages entre cités in Hérodote: V, 95; VI, 108; VII, 145, 154. Voir en général L. Piccirilli, Gli arbitrati interstatali greci, Pise, 1973, qui ne cite pas le cas milésien, car celui-ci fait référence à une situation de politique interne. 
ne conditionne donc pas la solution adoptée. Les Pariens n'agissent pas comme des arbitres, le terme diaıt $\alpha$ et ses dérivés n'apparaissent pas dans le texte d'Hérodote. Quand l'historiographie moderne parle de «l'arbitrage des habitants de Paros» en faisant référence à ce texte, elle omet qu'il existait réellement une institution d'arbitrage chez les Grecs, institution dont les caractéristiques ne sont pas présentes dans l'épisode qui nous intéresse et qui, commc nous le verrons plus bas, mérite attention pour notre travail. En effet, le récit souligne que les Pariens n'emploient que la vue, ils ne parlent à personne, ils visitent le territoire et écrivent, alors que l'arbitrage se caractérise par la parole, par l'obtention d'un arrangement verbal explicitement exprimé ${ }^{31}$.

D'autre part, bien qu'il soit difficile de préciser leurs sympathies

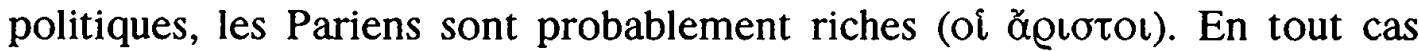
Paros se trouvait dans l'orbite politique de $\mathrm{Naxos}^{32}$. De plus, les conflits

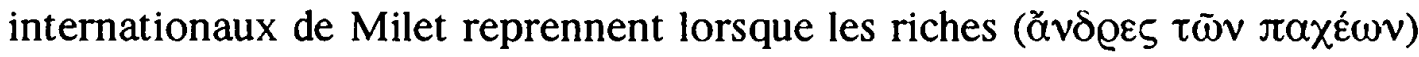
de Naxos sont expulsés par le démos et se réfugient à Milet, après que celleci a été rétablie par les Pariens et qu'Aristagoras en est devenu le gouvernant $^{33}$. En effet, celui-ci intercède devant le satrape Artaphernès pour qu'il envoie une expédition financée par les exilés contre Naxos. Bien que

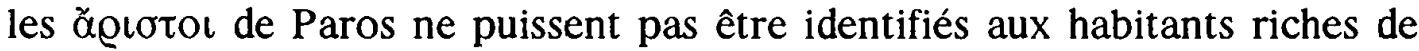
Naxos, il semble évident que le régime que rétablissent les premiers à Milet était le plus adéquat pour que les seconds en obtiennent de l'aide.

Il faut aussi considérer le fait que les Pariens se limitent à examiner les terres, les termes $\chi \omega \dot{\omega} \alpha$ et ày@òs apparaissent plusieurs fois dans le récit d'Hérodote. C'est-à-dire que leur geste prend seulement en compte les paysans de l'intérieur, à qui ils donnent la victoire, en opposition aux Milésiens vivant de la mer. De plus, il est fort possible que ces paysans soient les «populaires», au moins un certain nombre d'entre eux, que nous avons déjà vus et qui se situent dans la $\chi \omega ́ \varrho \alpha$ ou qui utilisent celle-ci comme base d'opérations. Dans ce sens, ils ne sont pas aussi neutres, du moins du point de vue sociologique, que ce qu'en dit Hérodote.

Le régime établi par les Pariens est une oligarchie présidée par des tyrans. Pour cela ils descendent des terres cultivées à la ville à fin d'expliquer la résolution qu'ils ont prise en adoptant le chemin inverse de celui des ácl-

31. Aristote, Rhétorique, I, 13, 1374 b 19-23, «il est équitable de préférer la parole et l'arbitrage».

32. Hérodote, V, 31. Robertson, art. cit., p. 377 signale, de plus, une communauté de culte aux Kabires entre Assèsos et Paros, ce qui préfigurerait la solution offerte par les médiateurs.

33. Hérodote, $\mathrm{V}, 30$. 
vaṽtal, qui prenaient leur décision en mer et revenaient au rivage pour la faire connaître à la cité. Ni la résolution des habitants de Paros, ni celle des åcı $\alpha \tilde{v} \tau \alpha$ d donnent lieu à une discussion. Les premiers lisent une liste de noms qui provient de leur «lecture» du territoire.

Ces actes trouvent un parallèle précis dans la manière d'agir du tyran Pisistrate à Athènes. Celui-ci faisait en sorte que les paysans se consacraient à leurs travaux et ne venaient pas résoudre leurs litiges à la citć, ainsi ils étaient plus prospères et étaient tenus loin des affaires publiques, tout en payant plus d'impôts du fait de leur enrichissement. Pour rendre cela institutionnel il créa des juges itinérants qui résolvaient les conflits entre les paysans grâce à l'arbitrage ${ }^{34}$. En définitive, les Pariens établissent un régime où les citoyens démobilisés sont gouvernés par le groupe réduit de la «liste», en opposition aux citoyens mobilisés que suscite Solon à Athènes, pendant la période archaïque aussi, lorsqu'il exige que les citoyens participent aux conflits sous peine d'à $\tau \mu i^{3} \alpha^{35}$.

D'autre part, ceux de cette «liste» partageaient des formes culturelles particulières que représente le poète milésien Phocylide. Il rejette de façon claire ce qu'implique la cité des ảeıvã̃ al ou de Thrasybule car «une petite cité qui est bien gouvernée au haut d'une montagne est plus forte qu'une Ninive insensée» ${ }^{36}$. De plus, en accord avec la décision des Pariens il recommande «si tu veux des richesses, cultive tes terres fertiles; car on dit qu'un champ cultivé est unc cornc d'Amalthée» (fr. 7). Bien sûr, il n'est pas étranger aux conflits et il défend l'éthique d'hetaireia: «l'ami doit parler avec l'ami des rumeurs qui courent parmi leurs concitoyens» (fr. 5). Ses aspirations ne sont pas du tout démocratiques, on doit intervenir loin de la lumière du jour et de l'assemblée: «prépare tes décisions la nuit: la nuit les hommes ont l'entendement plus clair, et la tranquillité est bonne pour celui qui cherche la vertu» (fr. 8). Plus précisément, un bon endroit pour conspirer avec les hetairoi la nuit est le banquet, dont il indique les normes dans le fr. 14. Finalement il soutient que dans la polis il préfère être «au milieu», car c'est le mieux. (fr. 12). Quand Aristote transmet ce passage il l'interprète du point de vue sociologique: Phocylide appartient à la classe moyenne $e^{37}$, mais le terme $\mu \varepsilon ́ \sigma o v$ a une valeur spatiale et politique claire. Il indique les

34. Aristote, Const. Athen., 16, 3-5. Hésiode recommandait déjà à son frère de s'abstenir de fréquenter la ville et de résoudre les conflits chez lui, Les trav. et les jours, 27-32, avec les analyses de B. Bravo, «Les Travaux et les Jours et la cité», Ann. Scuol. Norm. Sup. Pisa, 15, 1985, pp. 707-765.

35. Aristote, Const. Athen., 8, 5.

36. Fr. 4, numeration Rodríguez Adrados.

37. Politique, IV, 11, 1295 b 28. 
endroits où l'on prend des décisions ${ }^{38}$ et, d'après d'autres fragments de Phocylide (frs. 4,7), il pourrait représenter, de manière symbolique, les

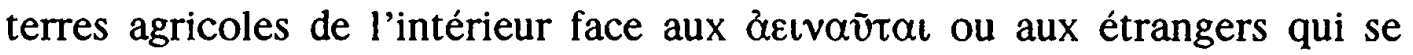
situent au-delà des frontières terrestres de Milet.

$\mathrm{Si}$ j'insiste sur la définition de ceux de la «liste», c'est parce qu'Anaximandre se trouvait dans le groupe qui avait gagné grâce à l'intervention des Pariens et qui formait cette liste bien que, assurément, Anaximandrc fat déjà mort. En effet, on connaît la liste des aisymnètes des Molpes depuis l'an $525^{39}$. Or jusqu'à la fin du siècle, le nom d'Anaximadre y apparaît trois fois comme patronyme de celui qui détient le pouvoir et le théonyme Mandros apparaît quatre autres fois dans des patronymes ou des prénoms. $\mathrm{Si}$ nous tenons compte de la forte tendance des normes onomastiques grecques à donner des noms en fonction des traditions familiales, il semble évident que ces Anaximandre seraient des parents du philosophe, de la génération de ses petits-enfants. Pendant la même période la terminaison ayogas apparaît quatre fois et parmi les noms il y a un Aristagoras. Il faut rappeler ici que les pères d'Histiée et d'Aristagoras s'appelaient précisément Lisagoras et Molpagoras (ce dernier est clairement en rapport avec les Molpes), de plus les tyrans étaient cousins et Aristagoras était le gendre d'Histiée ${ }^{40}$.

38. J.-P. Vernant, Mythe et pensée chez les Grccs. Études de psychologie historique. Nouvelle éd. revue et augmentée, Paris, 1985, pp. 216-219, 234-237, 247 248; M. Detienne, Les maîtres de vérité dans la Grèce archaïque, Paris, 1981 (1ère éd. 1967), pp. 83-98; P. Lévêque et P. Vidal-Naquet, Clisthène l'athénien. Essai sur la représentation de l'espace et du temps dans la pensée politique grecque de la fin du VIème siècle à la mort de Platon, Paris, 1964, passim.

39. L.H. Jeffery, The Local Scripts of Archaic Greece. A Study of the Origin of the Greek Alphabet and Its Development from the Eighth to the Fifth Centuries BC, Oxford, 1961, pp. 20-21 et 59 et sqq., rappelle qu'à l'inverse des usages en vigueur sous les grandes monarchies orientales, en Grèce on n'inscrivait pas les événements politiques des cités. Néanmoins il existe des listes de noms de magistrats et d'autres (champions olympiques) dont le but est chronologique. Elles étaient conservées par des experts en mémorisation des affaires publiques dont le nom a survécu même lorsqu'à la fin de l'époque archaïque ils commencèrent à employer l'écriture. La liste de Milet a été conservée ainsi jusqu'à ce qu'elle soit mise en écrit. D'autre part, nous rappelons que ce contexte de communication orale ou d'usage réduit de l'écriture favorise le fonctionnement oligarchique des institutions.

40. Hérodote, V, 30; P.L. Tozzi, La rivolta ionica, Pisa, 1978, pp. $97-99$ et 138140; Mazzarino, op. cit., p. 230. Je n'ignore pas la critique implicite que fait $F$. Bourriot, Recherches sur la nature du génos. Etude d'histoire sociale athénienne Périodes archaïque et classique, Lille-Paris, 1976, passim, à ce genre d'argument. Pour y répondre rapidement : d'une part il est vrai que l'historiographie moderne a 
Bien que la fonction de président des Molpes ne soit pas un poste politique, il est évident que les personnes qui l'occupent sont importantes du point de vue social et que parmi elles se situent les descendants d'Anaximandre et parents des tyrans de la cité. Les deux groupes se trouvent parmi ceux qui sont choisis par les Pariens dans leur liste de paysans aisés, peu mobilisés du point de vue politique et alliés fidèles des Perses $^{41}$. La révolte dcs Ioniens contre les Perses, commencée précisément à Milet par Aristagoras et Histiée, suppose un changement brusque de tendance sociologique et culturelle - ils récupèrent les positions de Thrasybule ou des ỏeıvaṽ $\alpha \iota$ - et se solde par un échec notoire. Les conflits internes de Milet vers le milieu du Vème siècle et leur résolution grâce à l'adaptation de modèles athéniens suivent la même ligne. En tout cas la cité est gouvernée de façon oligarchique, avec la différence que les groupes orientés vers l'Égée ont le soutien d'Athènes et son empire. Mais tout cela est déjà bien loin de la vie d'Anaximandre ${ }^{42}$.

\section{Ecritures d'Anaximandre}

Il semble donc fort possible que des descendants d'Anaximandre se trouvent dans la liste de paysans aisés de la $\chi \omega ́ \varrho \alpha$ milésienne établie par les ọ̌ı de Paros. Mais cette identification sociologique, avec toutes les réserves qu'elle présente, est-elle vraiment significative pour comprendre la production intellectuelle du philosophe?

J'essaierai de donner une réponse positive à cette question en la fondant sur l'examen du travail géographique d'Anaximandre et de son fragment. En effet, tous deux sont les témoignages les plus clairs d'une opération présentant des formes de communication spécifiques dont nous pourrons localiser les récepteurs et, ainsi, mieux comprendre le sens immédiat pour ceux-ci.

Il faut indiquer tout d'abord que les deux témoignages sont dissemblables. La carte de la terre représente l'espace en position statique en insistant, précisément, sur les notions d'équilibre, de stabilité, d'équidistance, etc; alors que le fragment explique les changements dans le temps. Ainsi,

rempli de manière incorrecte le contenu du terme, ce que Bourriot démontre amplement; d'autre part il faudrait étudier l'inexistence de liens de parenté en rapport avec lesquels sont montées des stratégies de pouvoir ou des tendances culturelles spécifiques, ce que Bourriot tend à minimiser; voir maintenant, F.J. González García, El Catálogo de las Naves. Mito y Parentesco en la Épica Homérica, Madrid, 1997.

41. Robertson, art. cit., pp. 376-379, insiste sur le fait que la liste représente l'oligarchie triomphante après les Pariens.

42. Ibidem, pp. 378-384 et passim, qui cite l'abondante bibliographie antérieure. 
Anaximandre explique quel doit être l'ordre de l'univers correct et, implicitement, par l'intermédiaire du vocabulaire et des termes employés, la position des hommes dans celui-ci. Étudions, donc, l'image du monde.

La représentation de la terre a été réalisée sur un $\pi i v \alpha \xi$ sur lequel était dessinée la totalité de sa superficie. Dès son origine, il ne s'agissait probablement pas d'une tentative de reproduction fidèle de la réalité, mais d'un objet destiné à être observé et contemplée ${ }^{43}$. Fn effet, il reflète une géographie qui sert, avant tout, à penser - et non pas à faire la guerre ${ }^{44}$. Le simple fait de fabriquer ce $\pi i v \alpha \xi$ est considéré comme un acte audacieux, propre aux hommes dont le courage est discutable ${ }^{45}$. En tout cas ce geste est immédiatement suivi par ceux d'imitateurs et d'usagers à Milet même. Hécatée perfectionne la carte et Aristagoras veut l'employer pour la guerre quand il essaie d'obtenir l'alliance militaire des Spartiates ${ }^{46}$. Pour sa part Hérodote se moque de ces cartes qui ne sont pas accompagnées d'un commentaire adéquat, qui représentent la terre tracée au compas entourée par Océan et donnent à l'Europe et à l'Asie des superficies semblables ${ }^{47}$.

43. Ch. Jacob, «Inscrire la terre habitée sur une tablette. Réflexions sur la fonction de la carte géographique en Grèce ancienne», in $M$. Detienne, (éd.), Les savoirs de l'écriture en Grèce ancienne, Lille, 1988, voir pp. 273-304, pp. 284-289.

44. Selon la formule d'Y. Lacoste, La Geografía un Arma para la Guerra, Barcelone, 1977.

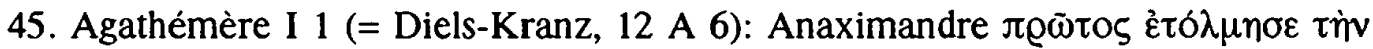

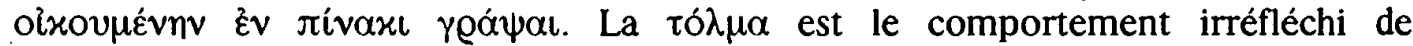

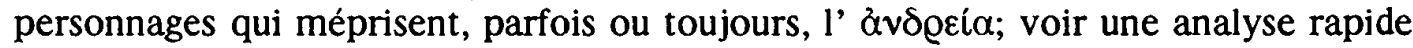
in M.V. García Quintela, «Esparta versus Tegea: entre la Leyenda y la Praxis Social», Gallaecia, 11, 1989, pp. 267-305, pp. 285-289. Le voile ayant comme décor les parties de la terre dont, selon Phérécyde, Zas fit cadeau à Chtonie lors de ses noces (Diels-Kranz, 7 B 2), doit être interprété comme partie d'une cosmogonie, mais bien que le voile brodé évoque un espace représenté, Phérécyde est resté dans le registre narratif; aucune tradition n'indique qu'il représente ses conceptions sur un plan. Ainsi, il faut penser à des représentations de l'espace comme celles de l'Iliade avec le bouclier d'Achille (XVIII, 483 et sqq., les premiers vers de cette description - 483-89 - évoquent une cosmologie) ou la broderie d'Hélène avec des scènes de la guerre de Troie (III, 125-128). Tout en laissant de côté les problèmes de rapport temporel avec Anaximandre.

46. Hérodote, V, 49 1, avec le commentaire de Lévêque et Vidal-Naquet, op. cit., pp. 80-83, et Jacob, art. cit., pp. 288-289.

47. Hérodote, IV, 362 , sans spécifier qui est l'auteur de la carte. Sur l'Océan dans les cosmologies grecques voir D. Fannini, «Okeanos. Dallo scudo di Achille alla mappa di Anassimandro", in A. Capizzi et G. Casertano (éds.), Forme del sapere nei presocratici, Rome, 1987, pp. 71-93. Voir aussi J. Rudhardt, Le thème de l'eau primordiale dans la mythologie grecque, Fribourg, 1971. 
On ne peut qu'être d'accord avec Hérodote, rien de tout cela n'est fidèle à la réalité. Mais il est évident que dans cette représentation l'Ionie, Milet si l'on veut, se situent à la frontière entre les deux continents et l'Océan le plus périphérique, elles sont donc ż $v \mu \varepsilon ́ \sigma \omega$, au centre du monde.

L'importance de cette notion de centre était déjà claire dans le fragment de Phocylide que nous avons cité plus haut, quand il signale que c'est la position qu'il préfère dans la cité identifiée aux paysans aisés. D'autre part, Thalès l'employait aussi dans ses projets politiques de résistance contre l'avancée des Perses, quand il suggérait de situer la capitale politique de l'Ionie unifiée à Téos, qui se trouvait $\dot{\varepsilon} v \mu \varepsilon ́ \sigma \omega^{48}$. De plus, cette notion n'est pas étrangère à

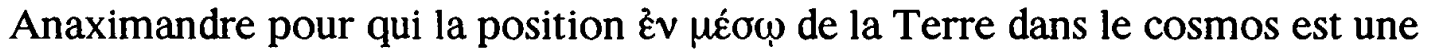
évidence ${ }^{49}$. Toutes ces données se trouvent bien documentées.

Nous pourrions transférer ces lectures au territoire de Milet tel que nous le connaissons d'après les témoignages examinés. Selon le point de vue adopté il pourrait y avoir deux centres et on peut imaginer une discussion politique sur celui qui serait le plus approprié. Le premier se situe à la

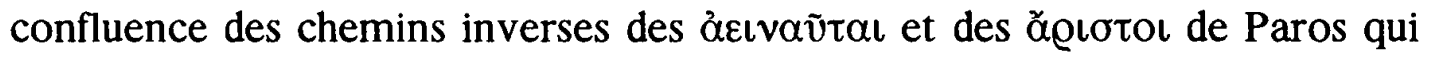
se déplacent de la mer ou de la $\chi \omega ́ \varrho \alpha$ avec les décisions politiques qu'ils ont

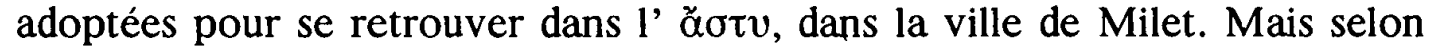
l'explication que donne Hérodote du partage du territoire milésien qu'ont réalisé les Perses après la défaite de 494 , le centre se trouve dans la plaine agricole d'où viennent les Pariens. Fn effet, on y distingue la cité et ses alentours, la $\chi \omega \dot{\varrho} \alpha$ agricole avec le temple d'Apollon à Didymes et les montagnes que les Perses cèdent alors aux Cariens ${ }^{50}$.

48. Hérodote, I, 1703 , voir supra n. 39.

49. Diogène Laërce II 1 (= Diels-Kranz, 12 A 1): «La terre est située au milieu ( $\mu \varepsilon \dot{-}$ бov), occupant le centre»; Hippolyte, Réfutation, I, 6, 3 (Diels-Kranz, 12 A 11): «La terre est suspendue librement, rien ne la gouverne, et elle est ferme à cause de sa distance semblable par rapport aux autres choses»; Aristote, Du ciel, II 13 295b (= Diels-Kranz, 12 A 26): Selon Anaximandre la terre «reste ferme à cause de son ó ótns... rien ne peut bouger plus vers le haut que vers le bas ou qu'en direction oblique, quand il est situé au $\mu \varepsilon ́ \sigma o v$ et reste $\delta \mu o i \omega s$ de la périphérie». Et voir le commentaire de Vernant, op. cit., pp. 216-237.

50. Hérodote, VI, 20 et supra $\mathrm{n}$. 15. Ce schéma se reproduit quand on considère la localisation des cinq dèmes dans lesquels se distribue le territoire milésien à partir du Vème siècle (Robertson, art. cit., p. 366) ou à partir de l'époque hellénistique (Piérart, «Athènes et Milet II», art. cit., pp. 295-296); tous deux suivent des analyses de L. Robert. Dans la périphérie, les Lérioi occuperaient les îles et les Teichiessê̂s, la frontière avec les Cariens à Teichioussa; la ville correspondrait aux Katapolítioi, le sud fertile de la péninsule avec Didymes reviendrait aux Argaseîs et le plateau central, entre Milet et Didymes, aux Plateîs. 
Dans ce contexte, deux offrandes que des enfants d'Anaximandre font au temple de Didymes au début du VIème siècle sont peut-être significatives ${ }^{51}$. En effet, au beau milieu d'une période de stasis à Milet, Anaximandre, qui appartenait à l'un des groupes en conflit (si c'était de forme autorisée ou non est sans importance, car il s'agit avant tout d'une relation symbolique), présente une image de la Terre dans le Cosmos et de Milet sur la Terre que l'on peut interpréter sans difficulté comme le résultat d'une extrapolation de sa propre localisation dans l'espace politique de la cité qui, ainsi, semble magnifié dans la mesure où il se situe żv $\mu \varepsilon ́ \sigma \omega ̣$.

Il faut souligner que l'objet, la carte sur le $\pi i v \alpha \xi$, sert à penser ${ }^{52}$. La preuve en est la réaction de Cléomène de Sparte, quand Aristagoras la lui montre. Mais la carte en elle-même ne veut rien dire. Elle a besoin d'une explication, ou bien verbale et complexe comme celle que donne Aristagoras, ou bien sous la forme d'une liste de noms géographiques ou ethniques qui convertit les superficies dessinées sans orientations immédiatement intelligibles en itinéraires praticables. Bien que nous ne sachions pas si Anaximandre a écrit cette liste, il est évident qu'Aristagoras en emploie une devant Cléomène et que le mot $\pi i v \alpha \xi$, qui décrit le support de la carte d'Anaximandre, sert aussi à désigner les supports sur lesquels s'établissent des listes destinées à être consultées $^{53}$. Mais, dans quel cadre cette consultation a-t-elle lieu?

Pour essayer de le définir il convient de souligner le changement d'attitude d'Aristagoras facc à l'asscmbléc des Athéniens. En effet, le Milésien emploie la carte et la liste seulement avec le roi de Sparte; face à l'assemblée des Athéniens, avec plus de succès, il utilise la rhétorique et évoque les liens qui unissent les Ioniens et les Athéniens ${ }^{54}$. L'inutilité de la carte dans le contexte de l'assemblée est mise en valeur par l'épisode des Nuées d'Aristophane

51. SylP, 3b; Tozzi, op. cit., p. 138; donnée qui insiste sur ce que nous avons déjà vu en ce qui concerne la localisation des Anaximandrides.

52. Contre Conche, op. cit., pp. 41-48, qui l'évalue comme un travail géographique réaliste.

53. Hérodote, IV, 36, 2, insiste sur la nécessité du commentaire comme complément de la carte. En V, 49, 5-7 Aristagoras récite un itinéraire (mémorisé) sur la carte, qu'Hérodote lui-même corrige en V, 52-54; au sujet de l'importance des itinéraires et des listes voir J. Goody, La raison graphique, Paris, 1979 (Cambridge

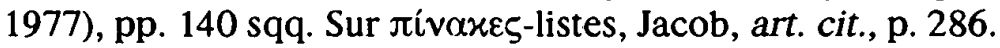

54. Hérodote, $\mathrm{V}, 97$. En plus du nombre différent des interlocuteurs d'Aristagoras et de leur localisation institutionnelle distincte, il est possible que l'emploi de la carte à Sparte puisse être mis en rapport avec des traditions au sujet d'un voyage d'Anaximandre dans cette ville (Diels-Kranz, 12 A 1, 5a) qui, selon G. P. Schaus, «Two notes on lakonian vases», Amer. Journ. Archaeol., 87, 1983, pp. 85-89, aurait laissé des traces dans l'iconographie de la terre qu'employaient des potiers lacédémoniens. 


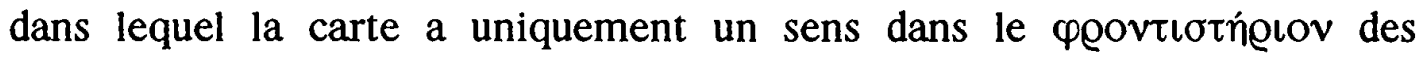
philosophes et est objet de dérision dans l'assemblée des spectateurs ${ }^{55}$.

La carte d'Anaximandre (et la liste qui existait probablement) ne pouvaient être communiquées qu'à un groupe restreint. Or ce groupe existe, il s'agit des hetairoi qui commentent les affaires de la cité dans les banquets, banquets qui étaient parfaitement institutionnalisés à Milet et qui étaient des endroits appropriés pour l'exposition de $\pi i v \alpha x \varepsilon \varsigma^{36}$. De plus, il faut souligner que le milieu social qui fournit la liste géographique (hypothétiquement d'Anaximandre ou celle qu'Hérodote fait exposer à Aristagoras), est celui que choisissent les Pariens dans leur liste et aussi celui qui forme la liste des aisymnètes des Molpes. Ce sont, donc, des processus intellectuels analogues qui limitent un groupe social spécifique.

Il est évident que la carte, même si elle est accompagnée d'une liste, a besoin d'une explication détaillée, d'un milieu social qui soit réceptif au commentaire fait par un notable ${ }^{57}$ et d'un contexte approprié pour cette communication. Essayer de comprendre la carte d'Anaximandre sans tenir compte de ces situations revient à nier l'existence de mécanismes complexes de formulation et de diffusion des connaissances et à admettre que la pensée fonctionne sans aucun type de limitations ${ }^{58}$. D'autre part, c'est cette explication orale nécessaire, qui va de pair avec l'information graphique, qui permet de lire le Cosmos et la Terre que présente Anaximandre comme la métaphore du territoire de Milet et de la localisation de sa famille dans celui-ci.

En tout cas, cette interprétation n'est pas obligatoirement celle d'Anaximandre. En effet, soutenir cette opinion impliquerait une connaissance approfondie de sa psyché, ce qui est, comme nous l'avons déjà dit, impossible. Mais, qu'il ait fait ce rapprochement de manière consciente ou non, dans le milieu social dans lequel il vivait, dans les contextes où ses idées

55. Aristophane, Nuées, 200-208.

56. Au sujet de Phocylide, voir supra et Hérodote, I, 146, 3.

57. Diogène Laërce, VIII, 70 (= Diels-Kranz, 12 A 8) recueille une information selon laquelle Anaximandre prenait un air tragique vêtu de cérémonie. La soulignent G. Colli, La sagesse grecque II, Combas 1991 (Milan 1978), ad loc. et Conche, op. cit., p. 27 n.6. Selon Colli, cet aspect facilite la communication. Le travail de S. Humphreys, «Transcendence and Intellectual Roles: The Ancient Greek Case», Daedalus, 1975, spring, pp. 91-118 est pionnier dans l'exploration des contextes sociaux de production et communication du savoir en Grèce Antique. L'œuvre de B. Betelheim et K. Zelan, Aprender a Leer, Barcelone, 1983 (New York, 1981), dont le sous-titre de l'édition originale «The Child's Fascination with meaning» est éclairant, m'a aussi fait réfléchir.

58. Voir J.C. Bermejo Barrera, «Ensayo sobre los Límites del Concimiento Humano», in Entre Historia y Filosofía, Madrid, 1994, pp. 7-43. 
pouvaient être communiquées, le rapprochement entre le monde du philosophe et celui de la réalité de Milet était viable et plausible ${ }^{59}$.

Si le cosmos tel que le dessine Anaximandre est une métaphore du territoire milésien gouverné par l'un des groupes qui l'habitent, qu'est-ce que peut indiquer le texte d'un fragment que l'on présente depuis l'Antiquité comme poétique et, donc, comme métaphore probable?

Il faut d'abord constater qu'Anaximandre n'est pas le seul de son époque à occuper une place ambiguë entre la prose et la poésie. Aristote dit la même chose de Phérécyde de Syros, qu'il situe dans une position intermédiaire entre les poètes antiques et les philosophes, qui «ne disent pas tout sous forme de mythe $»^{60}$. Dans le même sens Strabon énumère un groupe d'écrivains qui occuperaient une position intermédiaire: ils auraient abandonné la métrique mais conserveraient ce qui est propre de la poésie. Parmi eux se trouveraient Phérécyde, Cadmos, Hécatée et d'autres ${ }^{61}$. Il est fort probable que la critique qu'Héraclite fait d'Hésiode, de Pythagore, d'Hécatée et de Xénophane va dans le même sens. Comme ils essaient d'établir des formes de pensée plus synthétiques, ils tombent tous, d'une manière ou d'une autre, dans la conservation des procédés et des formes poétiques dont Héraclite considère qu'il faut les supprimer ${ }^{62}$.

D'un autre côté, cette forme de communication hybride exige, sans aucun

59. Le passage d'un dessin sur un canoë à des conceptions cosmologiques, et les intermédiaires nécessaires pour que l'ethnographe le comprenne correctement, fournit un parallèle intéressant, voir Ph. Descola, Les lances du crépuscule. Relations jivaros. Haute-Amazonie, Paris, 1993, pp. 265-266.

60. Aristote, Métaphysique, XIV, 4, 1091b 1-10.

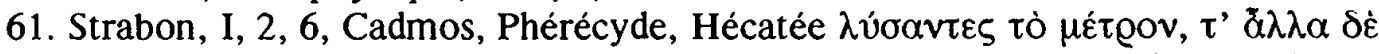

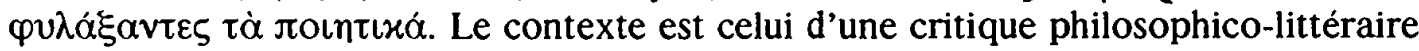
qui veut distinguer Homère en tant que géographe. Phérécyde a pris de l'importance dernièrement grâce à plusieurs travaux. Le premier, M.L. West, Early Greek Philosophy and the Orient, Oxford, 1971, pp. 1-75, a montré les relations des premiers présocratiques avec l'Orient; Colli, op. cit., II, pp. 77-104 et 273-279, a souligné le fond de sagesse et de mystique de la philosophie naissante; Kirk, in G.S.Kirk, J.E. Raven, et M.Schofield, The Presocratic Philosophers. A Critical History with a Selection of Texts ${ }^{2}$, Cambridge, 1983, pp. 50-71, reprend l'essentiel de Phérécyde dans la réédition du livre; enfin H.S. Schibli, Pherekydes of Syros, Oxford, 1990 , avec une édition utile de textes sur le personnage et une interprétation générale soignée.

62. Diels-Kranz, 22 B 40 commenté in M.V. García Quintela, El rey melancólico. Antropología de los Fragmentos de Heràclito, Madrid, 1992, pp. 89-93. Plus en général, L. Brisson, «Mythes, écriture, philosophie», in Mattéi (éd.), op. cit., pp. 4958 signale que la distance progressive entre les poètes et leur public permet l'apparition d'autres discours, comme l' «histoire» ou la «philosophie», qui s'expriment par l'intermédiaire de l'écriture. 
doute, un effort supplémentaire de la part des destinataires dans la mesure où elle ne s'ajuste pas aux modes de communication acceptés communément. Autrement dit, la prose, ou cette prose poétique dont se servent Anaximandre, Phérécyde, Hécatée et d'autres pour écrire sur la nature, les dieux et le monde est plus orientée vers la discrétion dans la communication que vers la quête de la rationalité qui, après tout, n'est rien d'autre qu'une étiquette moderne (dans la mesure où elle permet de se passer de ce qu'Havelock appelle le god apparatus ${ }^{63}$ ). Une nouvelle forme d'expression a besoin, de manière proportionnelle à son degré de nouveauté, d'un public très distinct, simplement rénové ou, au moins, semblable au consommateur des formes traditionnelles mais auquel on fait comprendre l'intérêt ou la nécessité de cette nouvelle forme. Comme le signalait justement $\mathrm{Ch}$. $\mathrm{H}$. Kahn, ce qui à long terme facilite la connaissance la rend difficile au moment de son élaboration.

Nous devons aussi rappeler qu'il y a des parallèles poétiques de l'emploi des métaphores avec des fins cryptiques ou discrètes. En effet, il existe des situations où l'on cherche des destinataires concrets dans une communauté. Il en est ainsi de la fameuse métaphore du navire/cité, qui présente de nombreux exemples dans la littérature grecque et qui est élaborée à partir de l'emploi de métaphores marines pour décrire le comportement des guerriers réunis dans l'Iliade. Chez Alcée on trouve l'un des premiers artifices de la métaphore: son contenu était destiné à la consommation restreinte des membres de son hétairie qui avaient l'habitude de se réunir dans des banquets. Un exemple particulièrement expressif est celui de Théognis de Mégare qui affirme explicitement qu'il s'adresse au groupe réduit de ceux qui sont dans le secret pour comprendre son analyse politique:

« maintenant nous allons à la dérive avec les voiles blanches repliées, au-delà de la mer de Mélos, au milieu de la nuit obscure; ils ne veulent pas écoper, la mer se lance sur les deux bords et il est difficile de se sauver ( $\sigma \omega \hat{\zeta} \varepsilon \tau \alpha \iota)$. Mais ils agissent: le timonier expert qui faisait une bonne garde a été éliminé, ils volent les marchandises violemment, l'ordre a disparu, le partage égalitaire n'existe plus $(\delta \alpha-$

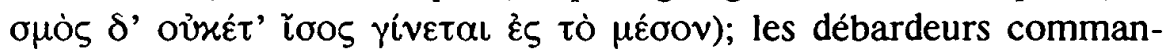
dent: aux bons les mauvais; je crains qu'une vague n'avale le navire. J'adresse ces énigmes dissimulées aux hommes de bien; celui qui est oọó 5 comprendra aussi le mal ${ }^{64}$.

63. E. A. Havelock, The Literate Revolution in Greece and Its Cultural Consequences, Princeton (NJ), 1982, pp. 229-232.

64. Theognis, 671-682, avec le commentaire de B. Gentili, Poesia e pubblico nella Grecia antica, Bari, 1984, pp. 58, 262 et tout le chapitre; Pindare aussi affirme que seules les personnes intelligentes peuvent comprendre sa poésie et que le reste a 
En définitive la prose poétique d'Anaximandre n'a pas été, ni n'a pu être, la seule chose qu'il ait élaborée. Elle a da être unie à une explication orale de ses contenus, probablement dans les milieux que nous connaissons déjà de participants aux banquets qui avaient des liens d'affinité sociale ou politique et qui étaient ceux qui comprenaient, ou à qui on expliquait de façon claire les contenus concrets des métaphores juridiques employées par Anaximandre. Deux textes nous montrent à quel point la communication fonctionnait ainsi.

Commençons par le Phédon de Platon, avec la déception de Socrate à la lecture du livre d'Anaxagore, déception qui le conduit à entreprendre sa deuxième «carrière» comme penseur qui caractérisera sa vie. Socrate entend quelqu'un qui dit qu'il disait que le voũs commande tout. Cela éveille en Socrate toute une série de résonances auditives sur le genre d'explication qu'il pensait trouver. Cela est aussi dá au fait que le langage employé était celui des hymnes de louange aux dieux, et en particulier à Zeus ${ }^{65}$. Mais Socrate refuse de suivre la voie de ces résonances qui, d'autre part, étaient connues des lecteurs d'Anaxagore. Il obtient le livre, lit et voit et sa déception vient, en fait, d'un usage équivoque des sens et d'une évaluation incorrecte de son rapport avec le genre de connaissance dont ceux-ci facilitent l'obtention. En effet, jusqu'au moment de la lecture, Platon se sert

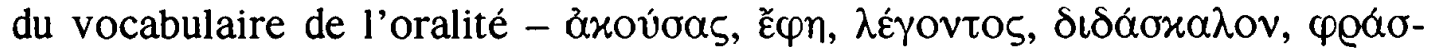
ocıv - alors que pendant et après la lecture il emploie le vocabulaire de la

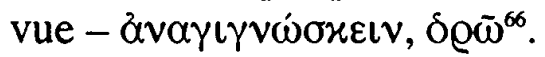

Ainsi, le livre d'Anaxagore n'explique pas tout. En plus d'exprimer formellement des idées par écrit, cette écriture évoque d'autres idées

besoin d'interprètes, Pindare, Olympiques, II, strophe V. Voir B. Gentili, «La giustizia del mare: Solone fr. 11 D., $12 \mathrm{~W}$., semiotica del concetto di díke in greco arcaico», Quad. Urb. Cult. Cl., 19, pp. 159-162 et Rocca-Serra, art. cit., pp. 81-82 à propos du besoin d'interprétation du sens caché de la poésie qui inspire l'exégèse allégorique.

65. K. Deichgräber, «Hymnische Elemente in der philosophischen Prosa der Vorsokratiker», Philologus, 88, 1933, pp. 347-361.

66. Platon, Phédon, 97c-99d (= Diels-Kranz, 59 A 47). Chez Platon les termes employés au sujet de l'activité intellectuelle viennent du vocabulaire de la vision, $L$. Paquet, Platon, La méditation du regard, Leiden, 1973. Brisson, art. cit., p. 53, souligne que pour Hérodote et Thucydide la vue est supérieure à l'ouïe dont ils tiennent compte. Au sujet des livres présocratiques voir Kirk, Raven, Schofield, op. cit., pp. 51-2 sur Phérécyde; pp. 102-3 Anaximandre; pp. 355-7, Anaxagore; West, op. cit., pp. 5-6; G. F. Nieddu, «Testo, scrittura, libro nella Grecia Arcaica e Classica: note e ossevazioni sulla prosa Scientifico-filosofica», Scrittura e Civiltà, 8, 1984, pp. 213-261; sur le livre d'Héraclite, García Quintela, op. cit., pp. 62-67. 
présentes dans la culture traditionnelle de ses auditeurs; pour comprendre le livre de manière adéquate, il faut tenir compte des échos culturels dans lequel il s'inscrit, échos dont Socrate rend compte sans les accepter. Des contextes de diffusion probablement restreints sont nécessaires ${ }^{67}$ ainsi que, sans aucun doute, une explication orale de la part de son auteur. ${ }^{68}$

Le second texte auquel je faisais référence se fait l'écho, au moins en partie, des problèmes que pose l'interprétation du livre d'Anaxagorc ct d'autres semblables. Plotin écrit:

«Ainsi les Anciens qui concordent le plus avec les enseignements de Pythagore et de ses disciples et de Phérécyde captent immédiatement sa nature [de l'Un]; mais alors que certains la travaillèrent dans leurs

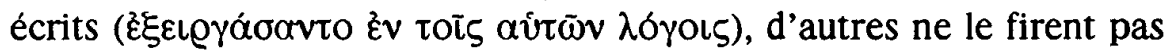

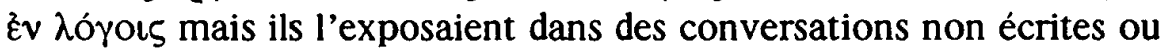

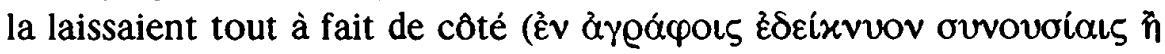
$\delta \lambda \omega \varsigma$ $\alpha \varphi \in \bar{\tau} \sigma \alpha v)\rangle^{69}$.

Pour comprendre les enseignements de tous ces premiers philosophes il fallait faire partie de leurs amis intimes ou de leurs disciples directs. En réalité, dans ces milieux restreints, on élaborait une pensée dont on mettait par écrit seulement de courts résumés, quintessence de réflexions longuement múries.

Pour en revenir à Anaximandre, nous savons que ces cercles plus restreints, aptes à la diffusion orale et écrite ${ }^{\prime \prime}$ (avec la manière particulière qu'adopte le Milésien) étaient bien identifiés dans sa cité et qu'Anaximandre s'y intégrait pleinement. Il n'était pas un marginal. De plus son cercle social et ses propositions ne sont pas démocratiques, tout au plus isonomiques, dans le sens propre à l'oligarchie que peut adopter ce terme ${ }^{71}$. Son groupe

67. La discussion d'Anaxagore avec le devin Lampon à propos d'une chèvre avec une seule corne, Plutarque, Périclès, 6 (= Diels-Kranz, 59 A 16), a un sens devant un nombre réduit de personnes : la dissection du crâne de l'animal faite par Anaxagore

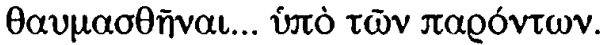

68. Aristote, face au livre d'Anaxagore, refuse aussi d'aller plus loin que le livre, Métaphysique, I, 985a 18-21.

69. Plotin, Ennéades, 5.1.9. 27-32.

70. R. Finnegan, Oral Poetry. Its Nature, Signifiance and Social Context, Cambridge, 1977, explique la complementarité d'écriture et d'oralité. J'essaie de montrer les conséquenccs dc ccla dans le cas qui nous occupe.

71. Je nuance ainsi Vernant, op. cit., pp. 216-237, en m'intéressant de plus près aux faits spécifiques de Milet. Pour le reste, les pages de Vernant servent de fondement à ces réflexions. Au sujet de l'isonomie, Mazzarino, op. cit., pp. 222-223 corrigé par Lévêque et Vidal-Naquet, op. cit., pp. 25-32. 
n'est rien d'autre qu'une partie de la cité qui aspire à la représenter dans sa totalité et probablement le fait après l'an 525. Ce groupe produit une culture spécifique dont l'exemple type est la poésie de Phocylide et, selon mon opinion, l'œuvre d'Anaximandre. L'écriture poétique d'Anaximandre et sa carte s'expliquent dans ce contexte. D'autre part, à cette époque, il existe d'autres exemples de l'emploi de la connaissance de l'écriture pour occuper des positions sociales prééminentes, dans la mesure où l'on sait employer cette technique comme instrument de pouvoir ${ }^{n}$. Lisons finalement le texte du fragment d'Anaximandre:

«A partir du moment où il existe génération pour les choses existantes, apparaît aussi la destruction selon la nécessité; car elles se rendent justice et paient peines mutuellement pour leur injustice, selon l'ordre du temps; en disant ainsi ces choses dans des termes assez poétiques»"

Nous ne nous intéresserons pas maintenant aux innombrables discussions au sujet des mots qui sont authentiques et de ceux qui reflètent l'influence de la transmission et à quel degré. Il faut de toute manière distinguer deux parties symétriques et de sens équivalent. Selon la plupart des commentateurs, le premier segment «A partir du moment où il existe génération pour les choses existantes, apparaît aussi la destruction selon la nécessité» est le plus contaminé par la transmission ou se borne à gloser le contenu des mots authentiques de la deuxième partie. De plus c'est dans cette première partie qu'il faut déduire une référence cosmologique ou cosmogonique, si l'on préfère. Ainsi, la lecture de ces références dans la seconde partie dépend exclusivement de l'acceptation d'un certain degré d'authenticité de la première et du contexte général de la transmission qui

72. Comme Méandre, le secrétaire de Polycrate de Samos, étudié par M. Detienne, «L'espace de la publicité, ses opérateurs intellectuels dans la cité», in Detienne (éd.), Savoirs, op. cit., pp. 29-81, pp. 73-81. Ou les privilèges qu'obtient le scribe Spensithios en Crète, voir F. Ruzé, «Aux débuts de l'écriture politique: le pouvoir de l'écrit dans la cité», in Detienne (éd.), Savoirs, op. cit., pp. 82-94, dont 83-84 avec bibliographie. Cela en marge de la polémique sur le degré de diffusion de la capacité de lire et d'écrire dans la Grèce archaïque.

73. Simplicius, Physique, 24, 18-20 (= Diels-Kranz, 12 B 1). Les discussions récentes les plus importantes sont Kirk, Raven, Schofield, op. cit., 117-122; Kahn, op. cit., pp. 166-193; H. Schmitz, Anaximander und die Anfänge der griechischen Philosophie, Bonn, 1988, pp. 26-35; Conche, op. cit., pp. 157-190. Soulignons la présence insignifiante d'Anaximandre dans le volume compilé par Mattéi, op. cit., cité 4 fois, sans être l'objet d'un examen spécifique, dans un livre de 438 p. 
dépend d'Aristote et de Théophraste et de leur compréhension des premiers phusiologoi ${ }^{74}$.

Dans la seconde partie, plus encore que le ton poétique, c'est le vocabulaire juridico-politique qui ressort. Dans ce sens, il est remarquable que la plupart des commentateurs du texte partent du principe incontesté qu'il s'agit de contenus physiques, philosophiques ou cosmologiques, comme on veut. Le vocabulaire employé est interprété comme la métaphore du processus de cette nature et, pour cela, on a recours ou bien aux interprétations des philosophes modernes, - en suivant le paradigme hégélien d'interprétation de la pensée avec et à partir de la pensée -, ou bien aux interprétations d'Anciens postérieurs: d'autres présocratiques et, surtout, d'Aristote qui, malgré tout, est toujours tenu en haute estime en tant que référence interprétative $e^{75}$.

Néanmoins, pour capter le sens plein du vocabulaire juridico-politique du fragment, on doit chercher les références dans des textes contemporains ou antérieurs, indépendamment de la tradition de pensée dans laquelle ils s'inscrivent, car l'autre option implique qu'il faut reconnaître qu'Anaximandre savait qu'il était en train de donner le jour à la philosophie. Supposition tout au moins aventureuse.

Si on laisse de côté ces considérations, dans la deuxième partie du fragment apparaissent trois idées fondamentales. En premier lieu, la nécessité d'un châtiment, de souffrir les effets de l'émission d'une sentence qu'exécutent les individus ou des groupes - car le vocabulaire juridicopolitique a, en définitive, une référence sociale (implicite si l'argument est cosmologique, explicite et explicable grâce au commentaire oral). En second lieu, l'injustice fonctionne comme moteur des alternances indiquées dans la première idée. Enfin, le processus est réglementé par une séquence temporelle définie.

74. Le scepticisme de Cherniss et McDiarmid (cités supra n. 1) est combattu par Kahn, op. cit., pp. 11-24; F. Solmsen, «Aristotle and Presocratic Cosmogony», Harv. St. Cl. Philol., 63, 1958, pp. 265-282; J.G. Stevenson, "Aristotle as Historian of Philosophy», Journ. Hell. Studies, 94, 1974, pp. 138-143. Il est évident que cette polémique est bien vivante.

75. Voir note antérieure. A. Capizzi, La repubblica cosmica. Appunti per una storia non peripatetica della nascita della filosofia in Grecia, Rome, 1982 essaie de construire une histoire de la philosophie antique non aristotélicienne, mais trop linéaire. Au sujet d'Anaximandre, pp. 299-311. Pour un examen des méthodes actuelles pour comprendre les présocratiques et le contraste avec la vision particulière des Grecs, voir G. Casertano, La nascita della filosofia vista dai Greci (morte o rinascita della filosofia?), Naples, 1977. 
La première idée évoque de forme précise les tribunaux de justice de la Grèce archaïque. Ce qui importait en eux étaient les parties présentes et leurs partisans qui étaient égaux juridiquement et, par conséquent, la réciprocité entre eux était viable et vraisemblable ${ }^{76}$. En effet, le juge ou arbitre qui arbitrait le conflit était d'un intérêt accessoire, à moins qu'il n'agît de façon claire pour son propre bénéfice" ${ }^{77}$. D'autre part, l'emploi du terme tíoıs s'intègre parfaitement dans les contextes judiciaires présents dans la poésie épique. Le terme tíoıৎ signifie alors le paiement que réclame l'offensé (exprimé de manière matérielle et symbolique) pour que sa $\tau \iota \mu n ́$ lui soit restituée ou soit augmentée, c'est-à-dire son prestige ou son honneur personnel et aussi ses propriétés. D'autre part, la qualité sociale des individus présents dans ces relations est toujours dissemblable. Bien qu'ils partagent le même code éthique hérö̈que, chacun doit être conscient de sa force relative et de sa position au moment de résoudre un conflit. Cette connaissance lui permettra d'agir de telle sorte qu'il récupère sa $\tau \iota \mu$ blessée ou, au contraire, qu'il se résigne à la perdre. Plus avant dans l'Archaîsme, le terme évoque la restauration de la $\tau \iota \mu$ ń qu'exigent des puissances divines pour compenser les injustices commises par les hommes, qui peuvent être parfois des fautes rituelles ${ }^{78}$.

Au contraire, l' ỏoıxía qui meut le processus se situe entre des égaux. Savoir supporter l'injustice est un précepte moral propre à l'aristocratie grecque, doté d'une vigueur spéciale dans le cas de Sparte, à cause de son archaïsme recherché. Comme dit P. Janni lorsqu'il analyse cette situation:

«Le regard du législateur se dirige moins vers le respect scrupuleux des droits de chacun que vers un possible affaiblissement de la vigueur morale qui assure le bien et la survivance de tous: autrement dit, une

76. P. Janni, La cultura di Sparta Arcaica, ricerche I, Rome, 1965, pp. 53-54. Cf. Théognis, 325-328 et 53-60 sur la réciprocité entre égaux dans la cité en tant que סíxn. Voir aussi Hésiode, Les trav. et les jours, 710-714.

77. L. Gernet, Recherches sur le développement de la pensée juridique et morale en Grèce (étude sémantique), Paris, 1917, pp. 137 et 459 et sqq. Exemples de juges injustes: Hésiode, Les trav. et les jours, 27-32 et supra n. 33; Théognis, 43-52. Ces cas semblent s'être généralisés et avoir donné lieu à des lois comme celle de Dreros (Crète), qui régit le temps d'exercice des magistratures pourvues de compétences judiciaires ou celle de Chios, qui établit la périodicité des réunions des organes judiciaires, voir H. van Effenterre et F. Ruzé, Nomima. Recueil d'inscriptions politiques et juridiques de l'Archaisme grec, I, Rome, 1994, pp. 306-309 et 262-267.

78. Solon 1, 25; Hymne Homérique à Déméter, 367; Janni, op. cit., pp. 73 et 71 et sqq. T.J. Saunders, Plato's Penal Code. Tradition, Controversy and Reform in Greek Penology, Oxford, 1991, pp. 19-20 et 46-47; A.W.H. Adkins, «Honour and Punishment in the Homeric Poems», Bull. Inst. Cl. Studies, 7, 1960, pp. 23-32. 
fois de plus on demande à l'individu qu'il se plie, qu'il accepte de souffrir l'injustice $(\hat{\alpha} \delta \iota x \varepsilon \bar{\tau} \sigma \alpha \alpha)$ qui peut le toucher dans la cohabitation avec ses égaux, pour ne pas menacer le bien précieux de cette concorde qui est la prémisse indispensable de la survivance du bon

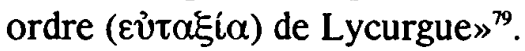

Il est évident que ce n'est pas le seul type d'injustice présent à l'époque. Il existe l'injustice entre des êtres de rang différent, comme celle qu'expriment les restitutions de $\tau \iota \mu$ qu'évoque le terme tíoıs. Cette forme d'injustice consiste à sortir de la place que chacun occupe dans la société. Pour l'idéologie sociale des Grecs, le bon ordre exige une société hiérarchisée où gouvernent les ặıơo et ce serait une injustice que ceux qui sont socialement inférieurs, xaxoí, prennent les rênes du pouvoir ou imitent les meilleurs $^{80}$. Dans des contextes érotiques il existe aussi une $\alpha \delta \iota x i \alpha$ semblable. Le terme exprime alors le fait que l'être aimé rejette les avances de l'amant. Si nous prenons en compte que la relation érotique chez les Grecs se produisait entre des personnes qui étaient dissemblables du point de vue social, ce rejet, cette $\not \dot{\alpha} \delta x i \alpha$ est la négation de la hiérarchie sociale ${ }^{81}$.

Dans cet ensemble de références, l' $\alpha \delta \iota x i \alpha$ d'Anaximandre est positive, elle permet de maintenir la cohésion sociale. Elle est nécessaire dans deux sens: du point de vue horizontal, à l'intérieur du groupe des ă@ıotoı qui sont égaux entre eux, pour les maintenir unis, en leur faisant oublier leurs querelles internes et respecter la $\tau \iota \mu$ ' de chacun d'entre eux; du point de vue vertical, face aux êtres inférieurs, pour exiger qu'ils continuent à occuper leur place sous peine de châtiment.

Finalement, l'expression d'un contrôle périodique est très fréquente dans toute sorte de textes constitutionnels ou para-constitutionnels de l'archaîsme grec. Des plus anciens, rhetra spartiate ou loi de Dreros, jusqu'aux plus modernes, lois de Chios ou poésie politique de Solon, on trouve toujours la mention de la périodicité des réunions des institutions civiques ${ }^{82}$. L'expres-

79. Janni, op. cit., pp. 53-54 et tout le chapitre II.

80. Solon, 24 D, 17-25; 25 D, 8-9. Cf. Alcman, 15 PMG.

81. Voir B. Gentili, «Historicidad de la Lírica Griega», in R. Bianchi Bandinelli (dir.), Historia y Civilización de los Griegos, II, Barcelone, 1982 (Milan 1978), pp. 64-147, pp. 80-84 et 128-129.

82. Voir supra n. 75. Solon, 24 D. souvent cité dans la discussion d'Anaximandre.

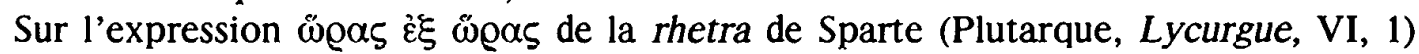
voir P. Oliva, Esparta y sus Problemas Sociales, Madrid, 1983 (Prague 1969), pp. $92-$ 93 qui apporte plusieurs hypothèses sur la périodicité des réunions. L'importance de la régulation du temps se trouve aussi au fondement du système de Clisthène, Lévêque et Vidal-Naquet, op. cit., pp. 22-24. 
sion du fragment d'Anaximandre est un corrélat précis de cet ensemble de situations qu'Aristote, plus tard, dans sa Politique, et non pas dans sa Métaphysique, corrobore en en faisant une notion abstraite ${ }^{83}$.

A partir de cela, le vocabulaire juridique permet deux interprétations solidaires du fragment. D'une part, c'est une métaphore descriptive des affrontements entre des groupes sociaux milésiens bien prouvés pendant la vie d'Anaximandre. D'autre part c'est une sentence prescriptive, elle indique comment les choses doivent se passer. Le fragment prétend organiser le monde de telle sorte qu'on évite le hasard comme résultat des crises. En ce sens, il s'agit d'une proposition positive. De plus, il faut souligner que ce devoir être s'oppose aux références historiques à la brutalité de la stasis milésienne. Cela signifie que le point de vue descriptif n'épuise pas tous les contenus du fragment. Cette constatation est importante si l'on cherche à savoir comment, à partir d'une perception du monde déterminée, on peut passer au registre cosmologique. Autrement dit, la réflexion sur la vie politique ou l'imprégnation de celle-ci sont des conditions préalables pour comprendre la nature qu'exprime les prétendus phusikoi milésiens ${ }^{84}$. Il n'existe pas de réflexion sur la nature isolée de ce qui l'entoure, comme dans un laboratoire scientifique moderne - dans lequel l'interaction avec le monde a aussi lieu, avec des mécanismes beaucoup plus indirects et sophistiqués.

D'autre part, dans une perspective diachronique ample, cette compréhension du Milésien n'est pas différente du projet platonicien de quête de la cité idéale à partir du rejet de la situation existente. Ainsi, la distance que Socrate exprimait dans le Phédon face aux phusikoi, conditionnée, comme nous l'avons vu, par le passage de la connaissance auditive à la connaissance oculaire, est plus rhétorique que réelle. Il se fonde plus sur un changement social réel et sur la modification des conditions de transmission de la connaissance que sur un changement véritable des sujets de réflexion et des propositions faites.

Mais, sans aller aussi loin dans l'évaluation de la sociologie de la pensée grecque, il y a un autre point qu'il faut souligner. Il s'agit de l'isolement historique des Milésiens. Il semble que Thalès, Anaximandre, Anaximène, Phocylide et Hécatée aient été incapables d'avoir des disciples: les héritiers de la physique milésienne se trouvent à Athènes; en effet, nous y trouvons

83. Politique, II 2, 1261 a 29 et sqq. Le contexte est la critique de la République de Platon.

84. Dans ce sens R. Mondolfo, En los Orígenes de la Filosofia de la Cultura, Buenos Aires $1960^{2}$ (lère éd. 1942), pp. 35-65 est un travail précurseur. 
Anaxagore, les atomistes, Diogène d'Apollonie et d'autres. Néanmoins, une révision rapide de l'histoire de Milet au Vème siècle montre que les tensions internes continuent en suivant les lignes de fracture existant au siècle antérieur, avec la différence que le rôle hégémonique extérieur passe de l'intérieur du continent à une puissance maritime et grecque: Athènes ${ }^{85}$.

Or, dans ces conditions d'instabilité politique et sociale, personne n'a-t-il pensé à partir des bases qu'avait établies Anaximandre?

\section{3. Échos d'Anaximandre à Milet}

Comme l'histoire de la philosophie traditionnelle présente la succession des idées dans le cadre qu'Aristote a défini comme propre à la physique antique, mis à jour selon la méthode proposée par Hegel, elle a refusé d'explorer les échos qu'ont pu avoir Anaximandre et son milieu culturel et social dans la cité qui l'a inspiré. En suivant la réflexion du Stagirite sur la pensée antérieure, on a cherché ses continuateurs dans la Métaphysique et non pas dans la Politique, comme je le suggère.

En effet, apparaissent deux théories constitutionnelles qui, tout en n'étant pas inspirées d'Anaximandre, s'inspirent tout de même de la cité de Milet, d'où sont originaires leurs auteurs respectifs. Nous assistons ainsi à une rénovation du processus de réflexion à partir de la réalité politique vers des formulations plus abstraites et de caractère directement politique. Sans l'intermédiaire d'une métaphore poético-cosmologique, propre à Anaximandre, la réflexion politique se renouvelle de manière autonome dans l'œuvre de deux Milésiens, Téléclès et Hippodamos, inégalement célèbres.

\subsection{Téléclès de Milet}

On connaît le personnage de Téléclès seulement par une mention isolée de la Politique d'Aristote ${ }^{86}$, ainsi, pour comprendre sa proposition il faut considérer l'argument du Stagirite. Celui-ci explique les trois parties qui forment une constitution et affirme que le bon legislateur doit prendre en considération : la délibération à propos des affaires communes, les magistratures, leurs pouvoirs et leurs dénominations, et l'administration de la justice. Puis il explique les sujets que considère la partie qui délibère (la paix, la guerre, le contrôle des magistrats etc.), en soulignant les modes propres aux démocraties et aux oligarchies. Plus particulièrement, dans le passage sur les modes démocratiques, il signale quatre possibilités qu'il ordonne selon le degré de démocratie et de participation ${ }^{87}$. Dans le passage qui se

85. Voir Robertson, art. cit., pp. 378-398, qui cite une bibliographie antérieure.

86. Cf. RE Pauly Wissowa s.v. Probablement postérieur à Hippodamos et antérieur à Aristote, il a pu être actif entre l'an 450 et l'an 350.

87. Politique, IV, 14, 1297b 35 sqq. 
réfère à la situation la moins démocratique il cite Téléclès :

«Que tous les citoyens décident sur toutes les questions est propre à la démocratie, car le peuple cherche ce type d'égalité, mais il y a plusieurs façons de comprendre ce «tous»: l'une des façons c'est qu'ils

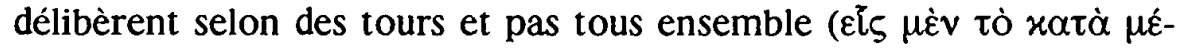

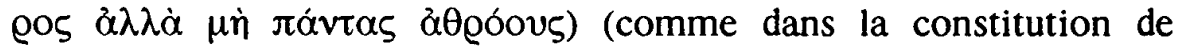

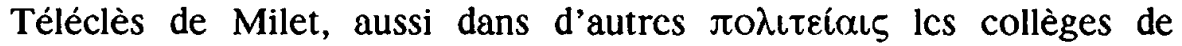
magistrats délibèrent en réunion, mais ils accèdent tous à la magistrature selon des tours, à partir des tribus et des divisions plus

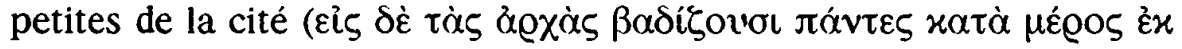

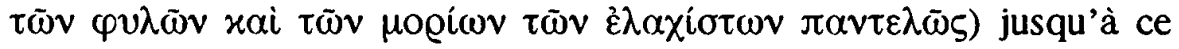
que les charges soient passées par tous), et qu'ils se réunissent tous seulement pour l'implantation de lois et pour les questions relatives au régime, et pour écouter les décrets des magistrats ${ }^{88}$.

Ainsi, le régime que préconise Téléclès, tout en étant démocratique, concorde, selon Aristote, avec des modalités moins démocratiques. De plus, le Stagirite est proche de l'esprit de ces normes quand, dans un autre contexte, il fait un éloge spécial de la règle de participation par tribus, qu'il présente comme un antidote aux changements et révolutions dans les régimes démocratiques ${ }^{89}$.

Dans le texte d'Aristote sont présents des échos de situations milésiennes connues, bien qu'il soit difficile d'établir les limites de la proposition de Téléclès, à cause de la rédaction. D'une part, à Milet, chaque fraction de citoyens délibérait toute seule. Dans les épisodes analysés plus haut, nous avons vu que les $\alpha \tilde{\varepsilon} \iota v \alpha \tilde{\tau} \tau \alpha \iota$ et les autres groupes en faisaient ainsi ${ }^{90}$. De plus, pendant la stasis, il n'existait pas d'institutions collectives qui prenaient des décisions, ou du moins on ne leur donnait pas de valeur. D'autre part, les sujets qui exigent les réunions collectives reproduisent comme un calque le procédé suivi par les ő@ıơo de Paros pour communiquer leur décision. Rappelons qu'ils convoquent une assemblée où ils annoncent une résolution de gouvernement et des normes relatives à la politeia, qui consistent à céder le pouvoir à quelques uns auxquels les autres ont l'obligation d'obéir.

88. Politique, IV, 14, 1298a 12-19.

89. $\mathrm{V}, 5,1305$ a 33 . Au sujet de cette forme politique qui présente les tribus comme les garants de la participation de toute la cité, ce qui n'est peut-être pas le cas pour ce qui nous occupe ici, voir F. Ruzé, "Les tribus et la décision politique dans les cités grecques archaïques et classiques», Ktema, 8, 1983, pp. 299-306.

90. Postérieurement il existe des vestiges épigraphiques prouvant cette situation quand les citoyens de différents endroits du territoire de Milet agissent avec un certain niveau d'autonomie, voir Piérart, arts. cit. 


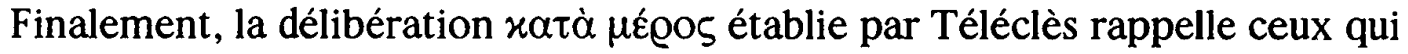

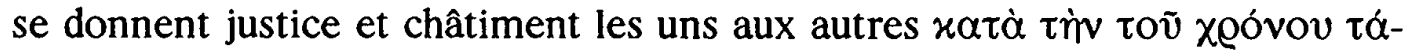
$\xi \iota v$ du fragment d'Anaximandre, car cette participation par morceaux ne peut avoir un sens qu'au fil du temps. Les segments de durée des mandats sont indissociables des segments de citoyens qui les exercent. De l'expression d'Anaximandre à celle de Téléclès ce qui change c'est seulement le point sur lequel on insiste, et non pas la chose en soi $^{91}$.

Bien qu'il soit impossible d'établir une filiation entre Anaximandre et Téléclès, il semble, néanmoins, évident que, malgré le peu de choses que nous savons de lui, on peut comprendre Téléclès dans le contexte milésien de primauté de formes de participation politique restreinte, ou de proposition de ces formes, que nous avons dégagé comme une constante.

\subsection{Hippodamos de Milet}

Aristote décrit aussi le régime politique imaginé par Hippodamos (Politique, II, 8), en signalant qu'il s'agit du premier théoricien politique ${ }^{92}$. Il projetait une cité de dix mille hommes divisés en artisans, agriculteurs et guerriers. La terre était aussi divisée en trois, une partie était consacrée aux dieux, une autre était communale, son usufruit revenait aux guerriers, et la troisième était exploitée par les agriculteurs. Le peuple, formé par les trois parties de la cité, élisait les magistrats qui s'occupaient des affaires communes, des étrangers et des orphelins. Entre autres, Aristote décrit une institution particulière du système d'Hippodamos:

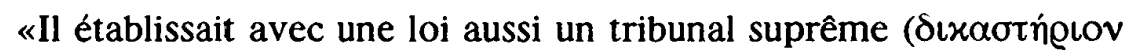
$\varepsilon v$ tò xú@ıov), auquel on devait remettre tous les procès qui semblaient mal jugés. En faisaient partie quelques anciens élus ( $\gamma \varepsilon \varrho o ́ v \tau \omega v$

91. Lévêque et Vidal-Naquet, op. cit., p. 23, expliquent le fonctionnement des prytanies à Athènes: «pour une tribu exercer la prytanie signifie à la fois occuper cette position au cours de l'année politique et déléguer cinquante des siens au foyer commun situé au cour de la polis».

92. La bibliographie s'occupe plus d'Hippodamos urbaniste que d'Hippodamos théoricien politique, qui est celui qui nous intéresse ici. Sa relation avec la pensée de la philosophie naissante a été évoquée par R. Martin, L'urbanisme dans la Grèce antique, Paris, 1956, pp. 104-106, dans la mesure où il lui attribue la conception du plan de Milet reconstruit en 479. Sa participation à ces travaux est remise en question par la révision chronologique du personnage faite par A. Burns, «Hippodamus and the Planned City», Historia, 25, 1976, pp. 414-428, qui situe sa naissance vers l'an 480 , et la constatation qu'aucune source ne lui attribue cette activité alors que sa participation aux travaux du Pirée, de Thourium ou de Rhodes concorde avec une chronologie plus récente. J'essaierai de montrer que sa relation avec Anaximandre peut être établie sur d'autres éléments. 
$\alpha i \varrho \varepsilon \tau \bar{\omega} v)$. En ce qui concerne les sentences des tribunaux, il croyait qu'on ne devait pas les voter avec des galets ( $\psi \eta \varphi$ ' plutôt en apportant chacun une tablette pour écrire la sentence ( $\varphi \dot{\varepsilon}-$

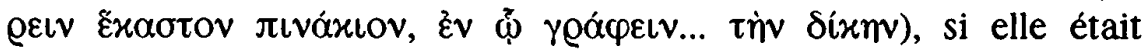
simplement condamnatoire; et elle serait laissée en blanc, s'il s'agissait d'un simple acquittement. Si elle condamnait et acquittait

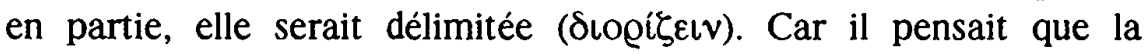
législation actuelle n'était pas bonne, car elle obligeait les juges à se parjurer lorsqu'ils dictaient l'une ou l'autre sentence»"

Aristote continue avec une critique de ces propositions, en les accusant surtout de confusion et il critique aussi, plus particulièrement, les tribunaux:

«La loi sur la résolution des juges n'est pas bonne non plus, ce n'est

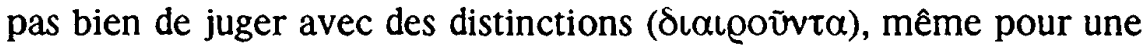

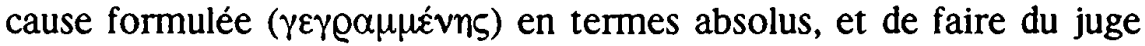

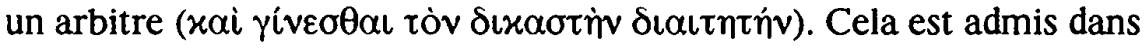

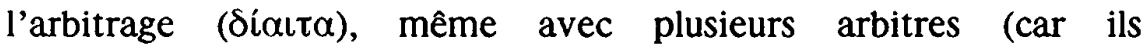
communiquent entre eux à propos de la décision); mais dans les $\delta\llcorner x \alpha-$ otń@ı $\alpha$ ce n'est pas possible, au contraire la plupart des législateurs établissent l'inverse: que les juges ne communiquent pas entre eux»"st.

Aristote signale la confusion qui provient des divergences entre les demandeurs et les juges et entre les juges eux-mêmes qui peuvent émettre

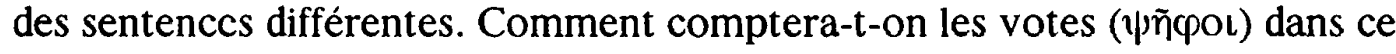
cas?

Il est évident qu'Aristote lit le projet constitutionnel d'Hippodamos du Vème siècle avec les yeux d'un analyste pointilleux des institutions juridiques athéniennes du IVème siècle. En effet, Aristote souligne les traits de la proposition d'Hippodamos qui inversent le sens des institutions athéniennnes connues. Ainsi, le tribunal suprême formé par les anciens élus inverse la situation des arbitres publics athéniens, qui sont tous les anciens dans leur cinquante-neuvième année désignés d'office pour résoudre les litiges en première instance, comme cela a été établi à partir de l'an $403^{95}$.

93. Politique, II, 8,1267 b $39-1268$ a 6.

94. Politique, II, 8, 1268 b $4-17$.

95. J'emploie consciemment un vocabulaire modernisant; vojir les nuances nécessaires, les explications plus détaillées et les discussions in R.J. Bonner et G. Smith, The Administration of Justice from Homer to Aristotle I, Chicago, 1930, pp. 349-355, II, 1938, pp. 97-116, 235-237; L. Gernet, Droit et société dans la Grèce ancienne, Paris, 1955, pp. 103-190; A.R.W. Harrison, The Law of Athens. Procedure, II, Oxford, 1971, pp. 64-68; le témoignage fondamental est celui d'Aristote, Const. Ath., 53. 
De plus, dans le procédé arbitral athénien, l'emploi de l'écriture était fondamental, car c'était en fonction de l'instruction menée par écrit que pouvait avoir lieu ou pas le recours en appel devant les tribunaux populaires

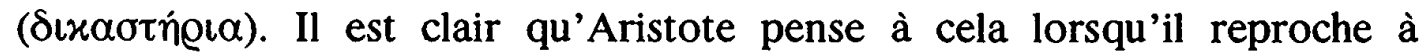
Hippodamos de proposer un arbitrage et non pas un vrai tribunal et de donner de l'importance à l'expression par écrit de la sentence.

Pour une fois Aristote est plus à l'aise dans le contexte évolué du droit athénien classique que dans des formes plus archaiques d'expression politique. En tout cas, bien que nous reconnaissions les confusions possibles du système d'Hippodamos, il est évident qu'Aristote ne fait pas d'efforts pour le comprendre. Cela est peut-être da au fait que le projet d'Hippodamos, comme l'œuvre d'Anaximandre, avait besoin d'une explication orale complémentaire, il est même possible que l'apparence excentrique qu'Aristote donne au personnage soit orientée dans cette direction: attirer l'attention et pouvoir mieux expliquer ses idées ${ }^{96}$.

Mais, si en acceptant le fond de l'analyse d'Aristote, nous nous trouvons face à une proposition d'une forme d'arbitrage, il ne faut pas comprendre celui-ci à partir de la perspective particulière du système athénien. En partant aussi de ce système, L. Gernet explique la philosophie profonde de l'arbitrage:

«...la notion de droit strict, à Athènes, n'a qu'une valeur relative; pour les tribunaux eux-mêmes, elle est une notion limite, l'idée force étant celle de dikaion, c'est-à-dire du juste; et c'est d'après le díkaion aussi que les arbitres sont tenus de se prononcer. Il reste qu'il est quelque chose de plus souple pour eux que pour la justice d'État. Il correspond à un sentiment du droit social - droit de la famille, droit du groupe local, etc. - qu'il appartient à l'arbitre de formuler et qui n'a pas nécessairement son écho dans le jugement d'un tribunal; parfois même, il peut y avoir opposition de principe, en tant que l'équité s'oppose au droit écrit: par exemple, dans la matière des successions, que la loi est loin de régler entièrement, il y a tout de même la loi, que les juges ne peuvent qu'appliquer; l'arbitre, lui, n'est pas tenu de juger suivant la loi. On pourrait dire que, dans toute conception du droit - mais c'est particulièrement vrai de la conception hellénique - il y a une synthèse entre les deux notions que

96. Politique, II, 8, 1267 b 24-28, et sa comparaison avec Diels-Kranz, 12 A 8 au sujet de l'apparence d'Anaximandre, voir supra n. 55. Ce genre de références, très abondantes et oubliées par les chercheurs modernes fait partie de l'anthropologie de la production intellectuelle dans la Grèce archaïque et classique qui n'a pas encore été écrite. 
les Grecs expriment par les termes d'aidōs et de díké: dans l'arbitrage, la première est la dominante.

Pour réaliser ce droit, c'est de la personne concrète de l'arbitre que l'on a besoin, et des rites caractéristiques de l'arbitrage. On n'admet pas facilement, on n'admet pas d'emblée, que le jugement des tribunaux de la ville, représentants d'une souveraineté abstraite, tranche les litiges privés.... En face d'une justice civique et impersonnelle qui s'impose a priori aux adversaires, qui tend à écarter les modes de preuve archaïques, qui a pour mission de faire prévaloir des normes législatives, qui règle en administrant, qui règle en une séance - en face de cette justice subsiste une méfiance instinctive qui est un trait de mentalité paysanne» ${ }^{97}$.

Il semble que le type de cité présenté par Hippodamos convient plus aux formes archaïques que l'arbitrage, tel que le décrit Gernet, actualise. Du moins ainsi semblent le montrer plusieurs éléments. D'une part le souci exclusif pour le statut de la terre et ceux qui en ont l'usufruit - en ignorant ce que représentait la mer pour des cités comme Milet et Athènes et en laissant de côté la position des artisans par rapport à la terre. D'autre part l'indication qu'il peut y avoir seulement trois causes, qui se réfèrent toutes à des affaires interpersonnelles: injures, dommages et morts; en ignorant la casuistique complexe du droit testamentaire, de propriété, commercial etc., qui est présente dans l'Athènes classique et qui, de certaine manière, a da être présente dans la société milésienne complexe du Vème siècle. Finalement, et ce n'est pas le moins important pour notre analyse, l'emploi de l'écriture dans des groupes restreints qui monopolisent les formes de pouvoir.

En effet, la lecture tendencieuse que fait Aristote rend insoluble un procès porté devant les tribunaux d'Hippodamos. Mais nous pouvons penser à une écriture de sentences préalable à une explication orale (chose qu'Aristote lui-même reconnaît nécessaire et propre, précisément, à l'arbitrage - xoıvo-

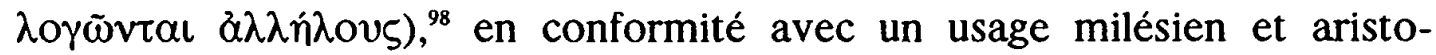
cratique que nous avons détecté dans la diffusion de la pensée d'Anaximandre et dans la médiation des Pariens. Dans ce cas nous nous trouvons devant une proposition institutionnelle en consonnance avec les formes restreintes de participation politique propres à toutes les formes de pensée émanant de Milet que nous avons eu l'occasion d'étudier. L'ambiance sociale et juridique que présente Hippodamos est celle d'une cité d'intérieur,

97. Gernet, op. cit., pp. 113-114.

98. Politique, II, 8,1268 b 7-8 et 10-11. 
avec une base agraire, où la prise de décision se restreint aux groupes des hommes armés ${ }^{99}$.

Or, à la fois socialement et politiquement c'était aussi la société d'Anaximandre et Phocylide. Si nous le prenons en compte, il convient de conclure avec quelques questions. D'úne part, n'est-il pas pertinent de chercher chez Téléclès et Hippodamos les authentiques continuateurs - non pas disciples ou partisans - d'Anaximandre, bien que dépourvus de poésie? D'autre part les Lois de Platon ne seraient-elles pas la culmination d'un processus de pensée politique de l'aristocratie grecque obligée de céder de plus en plus de parcelles de pouvoir et qui a pris la pensée utopique d'une cité agraire avec des contrôles minutieux des magistrats, nombre d'entre eux par écrit ${ }^{100}$, comme un authentique refuge intellectuel et moral, comme une espèce de placebo qui atténuait l'angoisse produite par une réalité qu'elle ne pouvait plus changer? Serait-il si difficile d'imaginer que le début de ce processus a lieu dans le fragment poétique d'Anaximandre, dans sa carte et son cosmos projetés à partir d'une perspective intéressée et partielle du territoire de sa propre cité? ${ }^{101}$

(Université de Saint Jacques

de Compostelle, Espagne)

Marco V. GARCIA QUINTELA

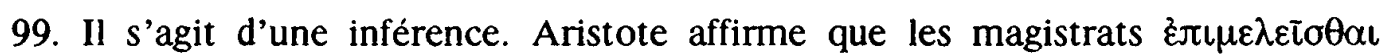

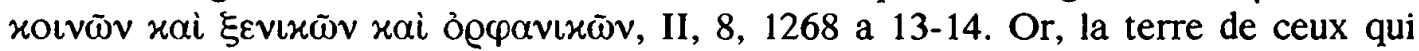
sont armés est, précisément, xoเví s'occuper des étrangers est toujours en rapport latent avec la guerre (cf. l'archonte polémarque à Athènes, Aristote, Const. Ath., 58) et les orphelins qui ont besoin d'aide seraient surtout les enfants de guerriers. En critiquant les magistrats, Aristote sous-entend que dans cette cité ceux qui portent des armes monopoliseraient les magistratures (II 8, 1268 a 21-25).

100. VI, $753 \mathrm{~b}$-d, analysé avec ses précédents et ses parallèles par M. Piérart, Platon et la Cité grecque. Théorie et réalité dans la Constitution des «Lois», Bruxelles, 1974, pp. 133-143, qui souligne de plus la rareté du vote par écrit dans la Grèce antique.

101. Mes remerciements à A. Ballabriga J.C. Bermejo, P. López Barja et M. Vegetti pour leurs commentaires apportés à une première version de ce travail. $M$. Torrevejano m'a donné l'occasion de présenter ces idées lors du séminaire sur Platon qu'il organise à l'Université de Valencia et dont les participants m'ont suggéré diverses questions que j'ai essayé de reprendre. Traduction de M.-P. Bouyssou. 